\title{
BCR-ABL RESIDUES INTERACTING WITH PONATINIB ARE CRITICAL TO PRESERVE THE TUMORIGENIC POTENTIAL OF THE ONCOPROTEIN
}

Pietro Buffa*1, Chiara Romano $\dagger^{1}$, Alessandro Pandini*, Michele Massimino $\dagger$, Elena Tirrò ${ }^{1}$, Francesco Di Raimondo†, Livia Manzella†, Franca Fraternali*² and Paolo G. Vigneri† $^{2}$

* King’s College London - Randall Division of Cell and Molecular Biophysics - London - UK

$\uparrow$ University of Catania - Department of Clinical and Molecular Bio-Medicine - Catania - ITALY

† Istituto Superiore di Sanità - Department of Hematology, Oncology and Molecular Medicine - Rome - ITALY

${ }^{1}$ These Authors contributed equally to this work

${ }^{2}$ Correspondence: Paolo Vigneri M.D., Ph.D.

University of Catania

Department of Clinical and Molecular Bio-Medicine

Via Androne, 85 - 95124 Catania - ITALY

Telephone: +39 (095) 312389

Fax: +39 (095) 7151928

E-mail: pvigneri@libero.it

Franca Fraternali, Ph.D.

King's College London

Randall Division of Cell and Molecular Biophysics

SE1 1UL - London - UK

Telephone: +44 (0) 207-8486843

Fax: +44 (0) 207-8486435

E-mail: franca.fraternali@kcl.ac.uk

Short title: Critical BCR-ABL residues determine TKI efficacy 


\section{ABBREVIATIONS}

Chronic Myeloid Leukemia (CML)

Dasatinib (DAS)

Imatinib Mesylate (IM)

Interleukin 3 (IL-3)

Kinase Domain (KD)

Kinase Inactive (KI)

Molecular Dynamics (MD)

Ponatinib (PON)

Tyrosine Kinase Inhibitor (TKI) 


\section{ABSTRACT}

Chronic Myeloid Leukemia patients that fail Tyrosine Kinase Inhibitors (TKIs) often present mutations in the BCR-ABL catalytic domain. We noticed a lack of substitutions involving four amino acids (E286, M318, 1360 and D381) that form hydrogen bonds with Ponatinib. We therefore introduced mutations in each of these residues either preserving or altering their physico-chemical properties. We found that E286, M318, I360 and D381 are dispensable for $A B L$ and $B C R-A B L$ protein stability but are critical to preserve catalytic activity. Indeed, only a "conservative" I360T substitution retained kinase proficiency and transforming potential. Molecular Dynamics simulations of BCR-ABL ${ }^{1360 T}$ revealed differences in both helix-alphaC dynamics and protein correlated motions consistent with a modified ATP binding pocket. Nevertheless, this mutant remained sensitive to Ponatinib, Imatinib and Dasatinib. These results suggest that changes in the four BCR-ABL residues described here would be selected against by a lack of kinase activity or by maintained responsiveness to TKIs. Interestingly, amino acids equivalent to those identified in BCR-ABL are conserved in $51 \%$ of human tyrosine kinases. Hence, these residues may represent an appealing target for the design of pharmacological compounds that would inhibit additional oncogenic tyrosine kinases while avoiding the emergence of resistance due to point mutations

Ponatinib, Imatinib and Dasatinib inhibit BCR-ABL ${ }^{\mathrm{I} 660 \mathrm{~T}}$ kinase activity suppressing its transforming potential

Key Words: $\quad$ CML, TKIs, Mutations, Resistance, Molecular Dynamics 


\section{INTRODUCTION}

Chronic Myeloid Leukemia (CML) has historically represented one of the few "paradigm diseases" in the field of cancer research (1-3). Indeed, CML has been one of the first malignancies associated with a known cytogenetic alteration (i.e. the Philadelphia chromosome) and a specific molecular hallmark (i.e. the BCR-ABL oncoprotein) $(4,5)$. Furthermore, specific BCR-ABL characteristics (e.g. constitutive tyrosine kinase activity, exclusive cytoplasmic localization) have been ingeniously targeted to devise innovative therapeutic strategies that have ushered the era of tailored cancer treatment $(6,7)$.

Suppression of BCR-ABL catalytic activity by the Tyrosine Kinase Inhibitor (TKI) Imatinib Mesylate (IM) has dramatically improved the natural history of CML (5). Despite the unparalleled results achieved by this drug, approximately 30\% of CML patients fail IM (3). To address this critical issue, second and third generation TKIs (e.g. Dasatinib and Ponatinib) have been generated and tested on IM-resistant individuals, enjoying early clinical success $(8,9)$. However, more mature data suggest that up to $50 \%$ of patients receiving either Dasatinib (DAS) or Ponatinib (PON) will not obtain long-term benefit from these drugs and will progress to the advanced phases of the disease $(10,11)$.

Multiple mechanisms have been proposed to explain TKIs failure. While this phenomenon can recognize different causes, point mutations in the BCR-ABL kinase domain (KD) are one of the most common reasons underlying TKI resistance (12-15). Substitution of Threonine 315 with an Isoleucine (T315I) represents the most problematic BCR-ABL mutation as alterations of this gatekeeper residue preserve the oncoprotein's kinase activity while inducing resistance to first and second generation TKIs $(16,17)$. Furthermore, recent evidence suggests that the T315I substitution is associated with inferior overall and failure-free survival (18).

Since the mechanisms allowing different BCR-ABL mutants to avoid catalytic inhibition by first, second and third generation inhibitors are still partially unresolved, there is a clear need for a structural characterization of the interactions occurring between the BCR-ABL KD and different TKIs. Experimental measures and quantification of the delicate balance of different physico-chemical components are very difficult to obtain and a complete overview of the molecular determinants in the inhibitors binding modes is challenging for this class of flexible proteins. Molecular Dynamics (MD) investigations represent an ideal tool to extract energetic and dynamical features in binding processes and conformational equilibria. In this respect, computational studies 
addressing BCR-ABL binding mechanisms can be grouped in three types of approaches: i) studies addressing the BCR-ABL KD alone, aimed at characterizing the equilibrium between the active and inactive conformations (19-21); ii) studies addressing binding to various inhibitors (22-24); and iii) studies evaluating the impact of mutations on BCR-ABL kinase activity (25-30). However, a comparative investigation of the binding modes and dynamics characterizing the interactions between the BCR-ABL kinase and different TKIs is still lacking.

By analyzing all reported BCR-ABL point mutations associated with TKI resistance (12-15), we noticed a lack of amino acidic substitutions involving four residues (E286, M318, I360 and D381) that are known to generate hydrogen bond interactions with PON (31) (Fig. 1A). The same residues (with the addition of T315) also generate hydrogen bonds with IM (32) (Fig. 1B), while T315 and M318 are the only amino acids forming hydrogen bonds with DAS (33) (Fig. 1C). We therefore employed a combined experimental and computational approach to investigate the effects of different mutations on these amino acids. Our results shed light on the molecular details underlying the essential role of E286, M318, I360 and D381 in preserving BCR-ABL kinase activity and oncogenic potential. We also outline some of the fingerprints of the inhibition mechanisms of PON, IM and DAS, offering a rationale for PON-dependent inhibition of all described BCR-ABL point mutations, including T315I. Since we report that $51 \%$ of human tyrosine kinases retain residues equivalent to those forming hydrogen bonds between BCR-ABL and PON, our results may aid the design of inhibitory compounds targeting additional kinases involved in the development of other malignancies. 


\section{MATERIALS AND METHODS}

\section{Mutagenesis and Constructs}

The $B C R-A B L$ sequence, cloned in the expression vector pcDNA3.1 (Life Technologies, Grand Island, NY, USA), was mutagenized using the QuikChange II XL Site-Direct Mutagenesis Kit (Agilent Technologies, Santa Clara, CA, USA). Incorporation of each mutation was verified by direct sequencing. Every $B C R-A B L$ construct was then amplified by PCR using the indicated forward (FLAG-tag underlined) 5'GACTAGTGCCACCATGGATTACAAGGATGACGACGATAAGATGGTGGACCCGGTGGGC-3' and reverse 5'-CGACGCGTCTACCTCTGCACTATGTCACT-3' primers and cloned in the SpeI and MluI unique sites of the pLEX lentiviral vector (Open Biosystems, Lafayette, CO, USA). To generate $A B L$ constructs corresponding to the reported native and mutated $B C R-A B L$, we amplified the first 425 base pairs of the $A B L 1 A$ coding sequence from HL-60 immortalized cells (DSMZ, Braunschweig, Germany) and substituted this amplimer to the $B C R$ sequences located upstream of the $A B L$ breakpoint. PCR reactions were carried out with the indicated forward (HA-tag underlined) 5'CTAGCTAGCGGATCCATGTACCCATACGATGTTCCAGATTACGCTATGTTGGAGATCTGCCTGAAG C-3' and reverse 5'-CTCAGCAGATACTCAGCGGCAT-3' primers. The ensuing PCR product was initially cloned in the pcDNA 3.1 vector using the NheI (on the forward primer) and $K p n \mathrm{I}$ (in the $A B L 1 A$ sequence) restriction sites. Each $A B L$ construct was subsequently subcloned in the BamHI and Not unique sites of the pLEX vector.

\section{Cell Culture, Transfection and Lentiviral Transduction}

$\mathrm{Ba} / \mathrm{F} 3$ cells (DSMZ) were maintained in RPMI-1640 medium (Sigma-Aldrich, St. Louis, MO, USA) supplemented with $4 \mathrm{mM}$ L-glutamine, $50 \mathrm{Units} / \mathrm{mL}$ penicillin, $50 \mu \mathrm{g} / \mathrm{mL}$ streptomycin (all from SigmaAldrich), 10\% fetal bovine serum (FBS) (Lonza, Basel, Switzerland) and 10\% WEHI-3B conditional medium, as a source of Interleukin 3 (IL-3). WEHI-3B cells (DSMZ) were grown in Iscove's Modified Dulbecco's Medium (Sigma-Aldrich), supplemented with $2 \mathrm{mM}$ L-glutamine, 50 Units $/ \mathrm{mL}$ penicillin, $50 \mu \mathrm{g} / \mathrm{mL}$ streptomycin, 10\% FBS and $25 \mu \mathrm{M}$ 2-mercaptoethanol (Sigma-Aldrich). All cells were grown at $37^{\circ} \mathrm{C}$ in $5 \%$ $\mathrm{CO}_{2}$. For the production of the lentiviral supernatant, transfer vectors (empty vector, native and mutant $A B L$ and $B C R-A B L$ constructs) were co-transfected with the packaging plasmids in the HEK293T cell line, using the calcium phosphate transfection method (Trans-Lentiviral ${ }^{\text {TM }}$ ORF Packaging Kit, Open Biosystems, catalog number: TLP4617). Viral supernatants were harvested 48 hours after transfection. Ba/F3 cells were then 
transduced by a double round of spin-infection (90 minutes, $1200 \mathrm{x} \mathrm{g}$ at $\left.32^{\circ} \mathrm{C}\right)$ in the presence of $1 \mathrm{~mL}$ of viral supernatant and $8 \mu \mathrm{g} / \mathrm{mL}$ polybrene (Sigma-Aldrich). Transduced cells were finally selected with $3 \mu \mathrm{g} / \mathrm{mL}$ puromycin (Sigma-Aldrich) for 72 hours.

\section{Proliferation Assays and Drug Treatments}

$1 \times 10^{4} \mathrm{Ba} / \mathrm{F} 3$ cells transduced with empty vector, wild-type or mutant $A B L$ or $B C R-A B L$ constructs were plated in quadruplicates in a 96-well plate in the absence of IL-3. Cell proliferation was assessed after 24, 48 and 72 hours using the ATPlite luminescence kit (Perkin-Elmer, Waltham, MA, USA) following the manufacturer's instructions. To calculate the $\mathrm{IC}_{50}$ values for cellular proliferation of different $\mathrm{TKIs}, \mathrm{Ba} / \mathrm{F} 3$ cells transduced with empty vector, wild-type, T315I and I360T BCR-ABL were plated in triplicate and incubated for 24 hours with logarithmic dilutions of PON (Santa Cruz Biotechnology, Santa Cruz, CA, USA), IM (a gift from Novartis, Basel, Switzerland) and DAS (a gift from Bristol-Myers Squibb, Princeton, NJ, USA). IL-3 was maintained in the culture media of $\mathrm{Ba} / \mathrm{F} 3$ cells transduced with the empty vector. Proliferation was measured using CellTiter96 Aqueous One Solution Reagent (Promega, Madison, WI, USA). IC 50 values were calculated using the logistic nonlinear regression included in GraphPad Prism 5.0 (GraphPad Software Inc.) and are reported as the mean of three independent experiments. For evaluation of total tyrosine kinase activity by immunoblots, $\mathrm{Ba} / \mathrm{F} 3$ cells transduced with $B C R-A B L^{\mathrm{WT}}, B C R-A B L^{\mathrm{T} 315 \mathrm{I}}$ or $B C R-A B L^{\mathrm{I} 360 \mathrm{~T}}$ were treated for 24 hours with the $\mathrm{IC}_{50}$ concentrations determined for PON, IM and DAS.

\section{Immunoblots}

$\mathrm{Ba} / \mathrm{F} 3$ cells expressing empty vector, native or mutant $\mathrm{ABL}$ or BCR-ABL proteins were starved of IL-3 for 4 hours (or 24 hours when exposed to TKIs) before centrifugation and suspension in isotonic buffer containing $100 \mathrm{mM} \mathrm{NaCl}, 25 \mathrm{mM}$ Trizma Base pH 8.5, $2 \mathrm{mM}$ PMSF and 1x protease inhibitor cocktail (Roche, Mannheim, Germany). Proteins were extracted by sonication and analyzed by SDS-PAGE loading $60 \mu \mathrm{g}$ of cell lysate per lane. Blots were probed using anti-HA (Covance, Princeton, NJ, USA), anti-FLAG (Sigma-Aldrich), anti-phosphotyrosine (clone $4 \mathrm{G} 10$ by Millipore, Billerica, MA, USA) and anti-Actin (Sigma-Aldrich) monoclonal antibodies.

\section{Purification of BCR-ABL proteins and in vitro kinase assays}

$B C R-A B L$ constructs cloned in the pLEX vector were modified to include six histidine codons at the N-terminal to allow protein purification. As a negative control, we generated a kinase inactive (KI) BCR-ABL mutant carrying the $\mathrm{K} 271 \mathrm{H}$ substitution ( $A B L 1 A$ numbering) in the ABL kinase domain as previously described (34). 
The $\mathrm{Ni}^{2+}$-nitrilotriacetic acid (Ni-NTA) Purification System (Life Technologies) was employed for protein purification under native conditions. $B C R-A B L$ constructs were expressed by transient transfection in HEK293T cells using the calcium phosphate method. After 48 hours, 1 x $10^{7}$ cells were lysed in $8 \mathrm{~mL}$ of $1 \mathrm{X}$ Native Binding Buffer, supplemented with 0.5\% Triton X-100, 5\% glycerol, $0.1 \mathrm{mM}$ PMSF and $2 \mu \mathrm{g} / \mathrm{mL}$ Aprotinin. Lysates were loaded onto pre-equilibrated Ni-NTA agarose columns and purified following the manufacturer's protocol. Proteins were then eluted in $6 \mathrm{~mL}$ of Native Elution Buffer and concentrated up to 100-fold by Spin-X UF Concentrators (Corning, Corning, NY, USA). Concentration of BCR-ABL proteins was determined by SDS PAGE analysis and Coomassie Blue staining using purified bovine serum albumin (BSA, Sigma-Aldrich) as a comparative standard, with quantification carried out by densitometry using the ImageJ software.

Detection of kinase activity was performed using the Kinase Glo luminescence assay (Promega) with an ABL peptide substrate (Abltide, SignalChem, Richmond, Canada). Assays were carried out in triplicates, at room temperature, for 1 hour, using $12 \mathrm{nM}$ enzyme, $50 \mu \mathrm{M}$ ATP and peptide concentrations of 1.56, 3.12, 6.25, 12.5, 25, 50, 100 and $200 \mu \mathrm{M}$. Luminescence values were measured using a Victor Wallac microplate reader (PerkinElmer) and normalized for background ATP to ADP conversion by omitting peptide substrate from the kinase reaction. Non linear regression fits (GraphPad Prism 5.0) were used to determine $\mathrm{V}_{\max }, \mathrm{K}_{\mathrm{m}}$ and MichaelisMenten kinetics by plotting velocity as a function of peptide concentration for each mutant. Velocity values are reported as signal to background ratio. $\mathrm{K}_{\mathrm{cat}}$ was obtained from the equation $\mathrm{k}_{\mathrm{cat}}=\mathrm{V}_{\max } /[\mathrm{enzyme}]$.

\section{Structures Preparation, Molecular Dynamics Simulations and Energetic Analysis}

Molecular Dynamics (MD) simulations were performed on the human wild-type ABL KD, and the T315I, I360T and I360R mutants. For the $\mathrm{ABL}^{\mathrm{WT}}, \mathrm{ABL}^{\mathrm{T} 315 \mathrm{I}}$ and $\mathrm{ABL}^{\mathrm{I} 600 \mathrm{~T}}$ systems simulations were also performed in complex with PON, IM and DAS. Starting structures for the mutants were derived by in silico mutagenesis with MODELLER v9.8 (35). The following structures were downloaded from the Protein Data Bank (36): inactive or "closed" conformation with PON (PDB: 3OXZ (31)), inactive conformation with IM (PDB: 2HYY (37)) and active or "open" conformation with DAS (PDB: 2GQG (33)). Unbound forms were generated by manual removal of the inhibitors. Missing residues were rebuilt with MODELLER v9.8. The GROMACS 4.0 program (38) was used to prepare the initial system coordinates, run the MD simulations, and analyze the resulting trajectories. Simulations were performed using the ffamber99sb (39) porting of the AMBER parm99 force field (40), the TIP3P model for water (41) and the General AMBER Force Field (GAFF) (42) with the AM1-BCC charges (43) for the inhibitors. The systems were minimized and then gradually heated to $300 \mathrm{~K}$ with potential restraints on the solute performing several steps of equilibration (44). After equilibration, the resulting structures were used as a starting point for production of $50 \mathrm{~ns}$ MD simulations. Secondary structure analysis 
was performed with DSSP (45). The extent of correlated motions was estimated from the correlation matrices on $\mathrm{C}^{\alpha}$ positions and represented as correlation webs (46). The energetic analysis was performed with the Molecular Operating Environment 2012.10 (MOE). The Pose Rescoring method of the Dock module was used to calculate the free energy of binding for each simulated system in complex with the three TKIs. Representative structures were extracted by cluster analysis (47) from each MD trajectory and validated with the MOE Structure Preparation module. Average values calculated over the representative structures of the three largest clusters were reported. The GBVI/WSA dG scoring function with the Amber12EHT force field was used (48). In silico mutagenesis scanning was performed with the Residue Scan method of the Protein Design module. The representative structure of the largest cluster in the MD trajectory of ABL ${ }^{\mathrm{WT}}$ was used. Sequence-based predictions of the effect of each mutation were performed with the web servers PROVEAN (49), SIFT (50) and polyphen-2 (51). Images were generated with VMD 1.8 .5 (52) and PyMOL 1.2 (Schrodinger Inc).

\section{Multiple sequence alignment of Tyrosine Kinase proteins}

Kinase domain sequences were downloaded from the website http://kinase.com. The human tyrosine kinase subset (90 sequences) was extracted and aligned using T-Coffee (53). 


\section{RESULTS}

PON binds to the inactive conformation of the BCR-ABL catalytic pocket by generating hydrogen bonds with four amino acids: E286, M318, I360 and D381 (Fig. 1A) (31). Interestingly, these residues do not undergo substitutions in patients failing TKI therapy (12-15). We therefore decided to generate point mutations in these sites and investigate their effect on the expression and kinase activity of both ABL and BCR-ABL. To this end, we engineered eight $A B L$ and eight $B C R-A B L$ constructs displaying two alternative substitutions in E286, M318, I360 and D381. The inserted mutations were deemed conservative if they preserved the physical/chemical properties of the amino acid found in the wild-type sequence, or non-conservative if they altered these characteristics (Table 1). $A B L$ and $B C R-A B L$ constructs displaying the wild-type sequence or bearing the T315I substitution were used as controls.

\section{E286, M318, I360 and D381 are dispensable for ABL and BCR-ABL protein stability but are critical to preserve BCR-ABL catalytic activity}

As point mutations in E286, M318, I360 and D381 have never been reported in CML patients failing a TKI, we hypothesized that substitutions in any of these residues might alter protein stability. To address this issue, we initially employed the above-indicated $A B L$ constructs that were lentivirally transduced in the $\mathrm{Ba} / \mathrm{F} 3$ cell line. We found that all $\mathrm{ABL}$ mutants were expressed at levels comparable to the wild-type protein and were devoid of catalytic activity (Fig. 2A) and transforming potential (Fig. 2B). Expectedly, the ABL ${ }^{\mathrm{T} 315 \mathrm{I}}$ control represented the only exception to these findings as this protein showed high kinase proficiency thereby allowing $\mathrm{Ba} / \mathrm{F} 3$ proliferation and survival in the absence of IL-3 (54) (Fig. 2A, $B$ ).

Since wild-type ABL displays very low tyrosine phosphorylation, we could not investigate the influence of each substitution in modulating catalytic activity. We therefore repeated the same experiments employing the corresponding $B C R-A B L$ constructs, exploiting the oncoprotein's constitutive kinase activity (2). While again all mutants were expressed at levels comparable to those of $\mathrm{BCR}-\mathrm{ABL}^{\mathrm{WT}}$, any substitution involving residues E286, M318 and D381 completely abrogated their kinase proficiency. The conservative I360T substitution was the only tested variation capable of maintaining BCR-ABL tyrosine phosphorylation (Fig. 2C). Accordingly, only $\mathrm{Ba} / \mathrm{F} 3$ cells expressing $\mathrm{BCR}-\mathrm{ABL}^{\mathrm{I} 360 \mathrm{~T}}$ survived and proliferated after IL-3 withdrawal, albeit at lower levels than those reported for BCR-ABL ${ }^{\mathrm{WT}}$ and BCR-ABL ${ }^{\mathrm{T} 315 \mathrm{I}}$ (Fig. 2D).

To further confirm these results we also performed an in vitro kinase assay using purified BCR-ABL mutants and a previously reported synthetic peptide substrate (55). The Michaelis-Menten parameters describing 
substrate binding $\left(\mathrm{K}_{\mathrm{m}}\right)$, maximum rate $\left(\mathrm{V}_{\max }\right)$ and catalytic constant rate $\left(\mathrm{K}_{\mathrm{cat}}\right)$ were determined using nonlinear regression fits. A KI BCR-ABL was included in the experiments as a negative control (34). As expected, seven of eight BCR-ABL mutants generated $\mathrm{V}_{\max }$ values similar to the KI protein, indicating complete lack of phosphorylation of the synthetic substrate (Fig. $2 E, F$ ). On the contrary, BCR-ABL ${ }^{\mathrm{I} 60 \mathrm{~T}}$ maintained its kinase activity, although at lower levels than $B C R-A B L{ }^{W T}$. However, the low $K_{m}$ value displayed by this mutant suggests that minimal substrate concentrations are sufficient to saturate its catalytic activity (Fig. $2 F$ ).

We complemented the experimental validation with an in silico screening of the expected effects of mutations on these positions (Table 2). Three sequence-based methods (PROVEAN, SIFT, polyphen-2) consistently predicted the majority of the substitutions as deleterious (labeled 'Not Tolerated' in SIFT or 'damaging' in polyphen-2). A few cases were predicted as deleterious by two of three methods and only selected substitutions in I360 were suggested as possibly tolerated. Sequence-based methods predict the expected effect on both stability and function. To isolate the contribution from protein structure destabilization we performed an analysis of the mutants using MOE. The difference in free energy of folding $(\otimes \otimes G)$ of the mutants versus the wild-type are also reported in Table 2. Substitutions on position M318 and I360 are generally destabilizing $(\otimes \otimes \mathrm{G}>1 \mathrm{kcal} / \mathrm{mol}$ in bold in Table 2$)$, whereas changes on E286 and D381 do not impact stability, suggesting that they are deleterious for their effect on function.

Overall, these results offer a solid rationale for the observed lack of mutations on residues E286, M318 and D381 in CML patients failing TKI treatment. Indeed, both conservative and non-conservative substitutions of these amino acids would result in loss of BCR-ABL catalytic activity, thus compromising its transforming potential. In contrast, a conservative substitution in position 360 appeared to preserve BCR-ABL kinase functionality.

\section{Molecular Dynamics simulations of BCR-ABL mutants I360T and I360R reveal differences in the helix- $\alpha \mathrm{C}$ dynamics and in the protein correlated motions}

In an effort to better understand the structural and dynamical differences between the KDs of the kinaseproficient I360T and the kinase-deficient I360R, we analyzed the MD trajectories of both BCR-ABL mutants and compared them to the wild-type oncoprotein. In particular, we investigated the correlation between the motions of the different parts of the protein. This was then mapped onto the protein structures using correlation webs, where residues that move together are connected by a line (Fig 3A, $B, C$ top panels). Correlated motions were previously shown to be critical for the activation of the ABL TK domain (26).

We found that the correlated motions of the BCR-ABL ${ }^{\mathrm{I} 60 \mathrm{~T}} \mathrm{KD}$ were mostly located at the bottom of the catalytic pocket. On the contrary the N-terminal of helix- $\alpha \mathrm{C}$ is not coupled in its motion to the N-lobe as in the 
wild-type domain. This provides more freedom of motion to helix- $\alpha \mathrm{C}$ that shifts upward and towards the outside (Fig. 3A, B, top panels). We then analyzed the conformational changes and stability of helix- $\alpha \mathrm{C}$ by plotting its time evolution along the sequence. We observed that, in BCR-ABL ${ }^{\mathrm{I} 600 \mathrm{~T}}$, helix- $\alpha \mathrm{C}$ maintained its structure despite retaining the modified orientation for the rest of the simulated time (Fig. $3 B$, bottom panel, red arrow). BCR-ABL ${ }^{\mathrm{I} 60 \mathrm{R}}$ presented a strong decrease in correlated motions compared to both the wild-type oncoprotein and the I360T variant (Fig. 3C, top panel). The I360R mutant also showed an upward shift of helix$\alpha \mathrm{C}$ that appeared to partially unwind for a $6 \mathrm{~ns}$ interval in the middle of the simulation (Fig. $3 C$, bottom panel, red arrow).

Overall, our results detected differences in the dynamics of BCR-ABL ${ }^{\mathrm{I} 360 \mathrm{~T}}$ and $\mathrm{BCR}-\mathrm{ABL}{ }^{\mathrm{I} 360 \mathrm{R}}$ that could be related to the diverse catalytic activity of these mutants. However, it remained to be established if different TKIs would successfully abrogate the catalytic activity of BCR-ABL ${ }^{\mathrm{I} 60 \mathrm{~T}}$.

\section{The persistence of some hydrogen bond interactions is critical for successful inhibition of $B C R-A B L^{I 360 T}$} kinase activity by TKIs

To investigate whether PON would suppress BCR-ABL ${ }^{\mathrm{I} 60 \mathrm{~T}}$ kinase activity we simulated this mutant's KD in complex with the drug, and analyzed the average distance of the residues involved in hydrogen bond interactions during the simulations. Since BCR-ABL ${ }^{\mathrm{I} 60 \mathrm{~T}}$ has never been reported in the literature, we also analyzed this mutant in complex with IM and DAS. Additional simulations were performed with the KDs of BCR-ABL ${ }^{\text {WT }}$ and BCR-ABL ${ }^{\mathrm{T} 315 \mathrm{I}}$ used as controls. The time evolution of the Root Mean Square Deviation on the $\mathrm{C}^{\alpha}$ atoms indicated that all systems rapidly reached equilibrium and remained stable during the simulated time (Supplemental Fig. S1).

PON binds the inactive conformation of the BCR-ABL ${ }^{\mathrm{WT}}$ kinase establishing four hydrogen bonds with E286, M318, I360 and D381 (31) (Fig. 4A). These interactions remained stable during the simulation with the exception of the transient binding with E286 (Fig. 4A). Since PON does not form hydrogen bonds with Threonine 315, its mutation to Isoleucine should not affect drug binding (56). Indeed, in our BCR-ABL ${ }^{\mathrm{T} 315 \mathrm{I}}$ PON system, the drug maintained persistent interactions with M318, I360 and D381, while again binding to E286 was transient (Fig. 4B). Moreover, PON's linear ethynyl linker helped the compound to skirt the steric hindrance phenomenon caused by $\mathrm{I} 315$. In the BCR-ABL ${ }^{\mathrm{I360T}}-\mathrm{PON}$ system, loss of the hydrogen bond with I360 coupled with the weak interaction with E286 did not affect the drug's ability to bind this mutant (Fig. 4C). Hence, our simulations suggested that PON should effectively suppress the catalytic activity of the I360T mutant. 
Like PON, IM binds the BCR-ABL KD in the inactive conformation generating hydrogen bond interactions with the four residues described above (E286, M318, I360, D381) plus the gatekeeper Threonine in position 315 $(32,57)$ (Fig. 4D). Our analysis showed that these interactions persisted during the entire BCR-ABL ${ }^{\mathrm{WT}}$ simulation, with small oscillations of the drug's tail that caused one short increase of the distance from I360 in the middle of the simulated time (Fig. 4D). In the BCR-ABL ${ }^{\mathrm{T} 315 \mathrm{I}}$-IM system, the drug adapted to the mutagenized catalytic pocket thanks to a slightly outward displacement, thus avoiding the increased steric hindrance caused by the Isoleucine side-chain. These changes can be appreciated by comparing the differences in IM structure depicted in Fig. $4 D, E$. This spatial rearrangement alleviated the clash of bulky residues. However, in the simulation, the drug lost three of five hydrogen bond interactions compromising optimal protein binding. Indeed, aside from the loss of the hydrogen bond with Threonine 315 because of its substitution with Isoleucine, we observed displacements of the interactions with E286 and I360 (Fig. 4E). Analysis of BCR$\mathrm{ABL}^{\mathrm{I} 360 \mathrm{~T}}$ in complex with IM revealed that all hydrogen bond interactions previously shown for the wild-type system were maintained over time, with the exception of the interaction with the mutagenized I360 residue. Insertion of a Threonine at this site caused a slight change in the conformation of the activation loop resulting in a missing hydrogen bond with the tail of the drug (Fig. $4 F$ ). However, IM binding appeared stable despite this alteration.

Unlike PON, DAS binds the BCR-ABL catalytic pocket in the active conformation forming three hydrogen bond interactions: one with T315 and two with M318 (33) (Fig. 4G). Hydrogen bonds analysis of BCR-ABL ${ }^{\text {WT }}$ in complex with DAS showed that these three points of contact were preserved over the entire simulation (Fig. $4 G$ ). Both biological and clinical evidences have demonstrated that DAS is unable to inhibit the T315I gatekeeper mutation $(58,59)$. Simulation of BCR-ABL ${ }^{\mathrm{T} 15 \mathrm{I}}$ in complex with DAS showed the expected loss of the critical interaction with $\mathrm{I} 315$ (33) (Fig. 4H). In this case the hydrophobic pocket of the BCR-ABL KD plays a key role in anchoring the phenyl ring of DAS through hydrophobic interactions with several amino acids. Four residues (M290, V299, I313 and F382) all located in this region (33), showed an increased distance from the inhibitor in the BCR-ABL ${ }^{\mathrm{T} 315 \mathrm{I}}$-DAS system, becoming unavailable for hydrophobic interactions (Supplemental Fig. S2). Thus, the loss of DAS binding to BCR-ABL ${ }^{\mathrm{T} 315 \mathrm{I}}$ can mainly be attributed to changes in the hydrophobic anchoring of the drug. However, DAS did not lose any hydrogen bond interactions with BCR$\mathrm{ABL}^{\mathrm{I} 360 \mathrm{~T}}$, implying effectiveness of this drug in inhibiting the kinase activity of the I360T mutant (Fig. 4I). In addition to the MD simulations, we also estimated the free energy of binding $(\otimes G)$ for each of the TKIs with the simulated systems. The $\otimes \mathrm{G}$ values were calculated with $\mathrm{MOE}$ and are reported in Table 3, together with their corresponding $\mathrm{p} K_{i}$ values. Results are generally consistent with our simulations: PON shows effective binding in all three systems, DAS has a decreased ability to bind BCR-ABL ${ }^{\mathrm{T} 315 \mathrm{I}}$, while IM maintains a strong 
binding to BCR-ABL ${ }^{\mathrm{I} 600 \mathrm{~T}}$. The apparent inconsistency of the values for IM:T315I and I360T:DAS can be explained considering that the approximate free energy calculation included in MOE cannot accurately describe contributions from significant conformational changes in the oncoprotein. From previous studies (54) we know that this is the case for the destabilization of the interaction of the gatekeeper mutant with IM. It is possible that conformational changes also play a role in modulating DAS binding affinity for the previously uncharacterized I360T mutant.

In summary, our computational analyses suggest that BCR-ABL ${ }^{\mathrm{I} 60 \mathrm{~T}}$ should be responsive to PON, IM and DAS.

\section{Ponatinib, Imatinib and Dasatinib inhibit BCR-ABL ${ }^{\mathrm{I360T}}$ kinase activity suppressing its transforming potential}

To ascertain the validity of our computational analysis on BCR-ABL ${ }^{\mathrm{I} 60 \mathrm{~T}}$ we initially exposed Ba/F3 cells expressing this construct to increasing concentrations of PON, IM and DAS after IL-3 deprivation. Calculation of the $\mathrm{IC}_{50}$ values for cellular proliferation in response to each TKI confirmed our computational predictions, as the $\mathrm{I} 360 \mathrm{~T}$ mutant was sensitive to each drug displaying inferior $\mathrm{IC}_{50}$ rates than the wild-type oncoprotein (Fig. $6 A, B$ and $C$, left panels, Fig. 6D). We next analyzed total tyrosine phosphorylation levels after a 24 hour exposure to the three inhibitors and found that BCR-ABL ${ }^{\mathrm{I} 60 \mathrm{~T}}$ catalytic activity was efficiently suppressed by each TKI (Fig. 5A, $B$ and $C$, right panels). Finally, our experiments also confirmed that PON was the only effective compound for the treatment of BCR-ABL ${ }^{\mathrm{T} 315 \mathrm{I}}$ (Fig. 5A), whereas the wild-type oncoprotein was responsive to all TKIs.

Thus, PON, IM and DAS all successfully inhibited BCR-ABL ${ }^{\mathrm{I} 60 \mathrm{~T}}$ oncogenic activity, as predicted by the hydrogen bond interactions analysis (Fig. 4). These data also explain why the I360T substitution has never been observed in CML patients unresponsive to TKIs as, in spite of the transforming potential of this mutant, BCR$\mathrm{ABL}^{\mathrm{I} 360 \mathrm{~T}}$ remains sensitive to multiple kinase inhibitors. 


\section{DISCUSSION}

Over 100 point mutations involving more than 60 amino acidic residues have been associated with TKI resistance in CML patients (12-15). However, no study has ever reported amino acidic substitutions involving E286, M318, I360 and D381, four residues of the BCR-ABL kinase that generate hydrogen bond interactions with PON (31). In this manuscript we present biological and computational data demonstrating that these four amino acids play a pivotal role in preserving BCR-ABL transforming activity. Indeed, our results suggest that with the exception of the I360T substitution - both conservative and non-conservative mutations in E286, M318, I360 and D381 abolish BCR-ABL kinase activity. These findings explain the lack of point mutations in these four amino acidic residues, as any change in these sites would be selected against by either lack of catalytic activity or maintained responsiveness to TKI treatment, as we observed with BCR-ABL ${ }^{\mathrm{I} 60 \mathrm{~T}}$. These findings are also corroborated by sequencing data derived from $95 \mathrm{CML}$ patients that either failed IM or achieved suboptimal responses according to the 2009 European Leukemia Net criteria (60). In these analyses we detected the E286K mutation in one subject, three substitutions at the M318 residue (M318T-V-I) in three patients, and three amino acidic replacements at the I360 residue (I360T-L-S) in five further patients. All substitutions were found in a small percentage of clones $(\leq 10 \%)$ and none were detected when sequencing the same patients at a later time (Stella S and Vigneri P, unpublished data).

Since the I360T mutation preserved BCR-ABL catalytic activity, we investigated this amino acidic substitution from a computational standpoint, comparing it to the corresponding non-conservative I360R mutation devoid of kinase activity. MD simulations of these two mutants revealed major differences in correlated motions and in the dynamics of helix- $\alpha \mathrm{C}$ as compared to wild-type BCR-ABL. I360T displayed strong correlations in the region between the $\mathrm{N}$ - and $\mathrm{C}$-lobes of the $\mathrm{BCR}-\mathrm{ABL}$ catalytic region. We also detected a significant upward shift of helix $-\alpha \mathrm{C}$, which is allowed more freedom and is partially displaced from the core of the domain, with an ensuing tendency in widening the access to the binding cavity. These results suggest a modified arrangement of the ATP pocket that could compromise ATP binding and would be compatible with the reduced kinase activity of this mutant. More dramatic changes were detected in the dynamics of helix- $\alpha \mathrm{C}$ in $\mathrm{BCR}-\mathrm{ABL}{ }^{\mathrm{I} 60 \mathrm{R}}$ as in this mutant the helix is looser and has a tendency to unwind. In addition, the domain has lost the inter-lobe communication known to be critical for activation (26). This is in line with the drastic reduction of kinase activity observed for this mutant. In addition, hydrogen bond interaction analyses followed by biochemical and survival assays demonstrated that PON, IM and DAS successfully inhibit BCR-ABL ${ }^{\mathrm{I} 60 \mathrm{~T}}$. 
Hydrogen bond analyses also contributed to explain the structural reasons underlying the resistance of the T315I gatekeeper mutation to IM and DAS. The common hypotheses advocated to explain IM and DAS failure in suppressing $\mathrm{BCR}-\mathrm{ABL}^{\mathrm{T} 315 \mathrm{I}}$ is that this substitution mediates drug resistance through a steric hindrance mechanism (61). However, by studying the dynamics of the inhibitor bound systems in-silico, we found that a slightly outward displacement of both IM and DAS allowed these drugs to adapt to the T315I catalytic pocket, thus circumventing the increased steric hindrance caused by the bulky Isoleucine side-chain (Fig. 4D, E). Indeed, the steric hindrance generated by the T315I mutation does not lead to complete obstruction of the binding cavity and the flexibility of the drug structure allows for a suitable rearrangement around the bulkier side-chain, unlike cases in which more rigid molecules cannot fit into partially (or totally) occluded pockets. This is generally not visible from a single experimental structure, but requires the simulation of the system to estimate the degree of flexibility of both drug and protein. We therefore investigated other mechanisms contributing to the lack of efficacy of IM and DAS on BCR-ABL ${ }^{\mathrm{T} 15 \mathrm{I}}$. In accordance with previous reports, we found that loss of hydrogen bonds between IM and E286, T315 (replaced by an Isoleucine) and I360 in the BCR-ABL ${ }^{\text {T315I }}$ KD played a pivotal role in IM failure against the T315I mutation (30). However, loss of hydrogen bonds was not sufficient to explain BCR-ABL ${ }^{\mathrm{T} 151}$ resistance to DAS as only the interaction between T315 and DAS was compromised in the T315I mutant simulations. Upon further investigation we detected a perturbation in the dynamics of amino acidic residues that form hydrophobic interactions with the drug. These alterations are likely to factor heavily in the lack of DAS binding to the T315I mutant.

Further computational investigations of BCR-ABL ${ }^{\mathrm{T} 315 \mathrm{I}}$ revealed a net enhancement of correlated motions within the $\mathrm{N}$ - and C-lobes as compared to the wild-type oncoprotein (Fig. 6A, B). The mutation affects the dynamics of the two lobes modifying the access to and the shape of the binding site. The correlations also involve helix- $\alpha \mathrm{C}$ (which undergoes a partial unwinding) and the activation loop, suggesting that the T315I mutation dynamically perturbs several structural elements crucially involved in drug binding (Fig. 6B). This observation is in line with previous suggestions about the main role of gatekeeper mutations in orchestrating an effective communication between the two lobes (26) and in stabilizing the hydrophobic spine $(17,54)$. Simulation of the BCR-ABL ${ }^{\text {T315I }}$ PON system showed that, aside from the effects ascribed to the ethynyl linker in bypassing the steric hindrance (31), three pivotal features enabled this drug to inhibit BCR-ABL ${ }^{\mathrm{T} 315 \mathrm{I}}$ : i) as in the wild-type domain, PON showed intermittent interactions with $\mathrm{E} 286$, which is often dislocated from its natural position when helix- $\alpha \mathrm{C}$ is destabilized; ii) the drug maintained the same hydrogen bond interactions and the same average distance with all residues involved in hydrophobic contacts in the BCR-ABL ${ }^{\mathrm{WT}}$-PON simulation; iii) there were only minor differences between the BCR-ABL ${ }^{\mathrm{WT}}-\mathrm{PON}$ and BCR-ABL ${ }^{\mathrm{T} 15 \mathrm{I}}-\mathrm{PON}$ systems in terms of correlated motions 
(Fig. 6C, D), implying that PON seems to "revert" the dynamics of the T315I mutant to those of BCR-ABL wild-type.

The critical issue stemming from our results concerns the implications of the reported findings for future therapeutic developments. CML patients unresponsive to TKI treatment sometimes develop point mutations involving multiple residues within the BCR-ABL KD $(12-15,62)$. It should be emphasized that amino acidic substitutions engaging different residues of a catalytic domain are not a prerogative of the ABL kinase, as identical phenomena (including mutations of the gatekeeper Threonine) have been reported in patients diagnosed with lung cancer or gastro-intestinal tumors failing Epidermal Growth Factor Receptor or c-KIT inhibitors, respectively $(63,64)$. Two different approaches have been pursued to address these problems. Several companies have synthesized irreversible TKIs that, unlike reversible inhibitors (e.g. IM), generate covalent bonds with specific amino acidic residues (usually Cysteines but also Serines, Threonines or Lysines) of their substrate(s). While highly effective, irreversible TKIs have often been associated with increased (sometimes limiting) toxicities that have hindered their clinical development (65). An alternative approach has been to design compounds that do not interact with the critical gatekeeper residue of the substrate kinase (66). The caveat of this method is that - theoretically - amino acidic substitutions in residues other than the gatekeeper could induce resistance to these drugs. However, to date, no single point mutations have been associated with resistance to PON. Our data suggest that the lack of mutations causing PON failure derives from the fact that this inhibitor forms hydrogen bonds with four critical residues (E286, M318, I360 and D381) of the BCR-ABL KD that, if mutated, would compromise the transforming ability of the oncoprotein. Hence, changes in any of the above-mentioned four residues would be selected against by a lack of kinase activity or by maintained responsiveness to TKIs (BCR-ABL ${ }^{\mathrm{I} 600 \mathrm{~T}}$ ). Interestingly, when we analyzed the twelve known PON biological targets $(9,67)$, we found that these tyrosine kinases conserved the ABL E, I/V and D residues but displayed different substitutions in place of M318 (Fig. 7A). These results suggest a structural scenario in which E286, M318, I360 and D381 are "untouchable" residues for the ABL kinase, but only E, I and D are critical for the remaining PON targets as the Methionine in position 318 can be replaced by either an Alanine or a Cysteine.

Remarkably, residues equivalent to ABL E286, M318, I360 and D381 are conserved in 46 of 90 (51\%) human tyrosine kinases including both receptor and non-receptor proteins (Fig. 7B). Forty-two of these 46 tyrosine kinases were tested for PON responsiveness in a previously published in vitro kinase assay and 25 of 42 (59\%) were efficiently inhibited by the drug $\left(\mathrm{IC}_{50} \leq 20 \mathrm{nM}\right)$ (9). While we can't offer a detailed explanation as to why PON lacks efficacy on the remaining 17 kinases, several observations should be kept in mind. Specifically: i) 
while a sequence alignment suggests that E, M, I and D residues are preserved in these TKs, a large-scale Molecular Dynamics study would be necessary to confirm that these amino acids conserve a similar role in protein flexibility compared to the $\mathrm{ABL} \mathrm{KD}$, in line with recent evidence that selective inhibition of TKs could also be connected to differences in their intrinsic dynamics $(19,22)$; ii) since our current structural information is mostly limited to the catalytic domain of multiple tyrosine kinases, we can not account for the significant modifications that other portions of the protein may exert on the catalytic pocket, heavily affecting PON binding; iii) likewise, the shape and orientation of the drug binding pocket of each TK may present subtle differences that could partially or completely abrogate its interaction with PON. Hence, preservation of E, M, I and $\mathrm{D}$ residues in positions equivalent to those of the $\mathrm{ABL} \mathrm{KD}$ appears to be necessary but not sufficient to predict PON binding to other TKs.

In summary, we report here the identification of four amino acidic residues (E286, M318, I360 and D381) that are critical for BCR-ABL catalytic activity and leukemogenic potential. We also find that these amino acids are conserved in several human tyrosine kinases and are likely to represent unmodifiable residues that contribute to the proper structural folding and enzymatic activity of these proteins. Small molecule inhibitors targeting these amino acids in other tyrosine kinases might be of great clinical interest to avoid the emergence of resistance due to point mutations. 


\section{ACKNOWLEDGMENTS}

We thank Maria Stella Pennisi, Stefania Stella and Silvia Rita Vitale for critical reading of the manuscript. This work was supported by an investigator grant to P.V. from Associazione Italiana per la Ricerca sul Cancro (AIRC) and by funding from the Biotechnology and Biological Sciences Research Council (BB/I023291/1 and BB/H018409/1 to AP and FF). P.B. is the recipient of an AIRC - Marie Curie fellowship. 


\section{REFERENCES}

1. Goldman, J. M., and Melo, J. V. (2003) Chronic myeloid leukemia--advances in biology and new approaches to treatment. $N$ Engl J Med 349, 1451-1464

2. Melo, J. V., and Barnes, D. J. (2007) Chronic myeloid leukaemia as a model of disease evolution in human cancer. Nat Rev Cancer 7, 441-453

3. Deininger, M., O'Brien, S. G., Guilhot, F., Goldman, J. M., Hochhaus, A., Hughes, T. P., Radich, J. P., Hatfield, A. K., Mone, M., Filian, J., Reynolds, J., Gathmann, I., Larson, R. A., and Druker, B. J. (2009) International Randomized Study of Interferon Vs STI571 (IRIS) 8-Year Follow up: Sustained Survival and Low Risk for Progression or Events in Patients with Newly Diagnosed Chronic Myeloid Leukemia in Chronic Phase (CML-CP) Treated with Imatinib. ASH Annual Meeting Abstracts 114, 1126-

4. Kantarjian, H. M., Talpaz, M., Giles, F., O'Brien, S., and Cortes, J. (2006) New insights into the pathophysiology of chronic myeloid leukemia and imatinib resistance. Ann Intern Med 145, 913-923

5. Druker, B. J. (2008) Translation of the Philadelphia chromosome into therapy for CML. Blood 112, 4808-4817

6. Druker, B. J., Tamura, S., Buchdunger, E., Ohno, S., Segal, G. M., Fanning, S., Zimmermann, J., and Lydon, N. B. (1996) Effects of a selective inhibitor of the Abl tyrosine kinase on the growth of Bcr-Abl positive cells. Nat Med 2, 561-566

7. Aloisi, A., Di Gregorio, S., Stagno, F., Guglielmo, P., Mannino, F., Sormani, M. P., Bruzzi, P., Gambacorti-Passerini, C., Saglio, G., Venuta, S., Giustolisi, R., Messina, A., and Vigneri, P. (2006) BCR-ABL nuclear entrapment kills human CML cells: ex vivo study on 35 patients with the combination of imatinib mesylate and leptomycin B. Blood 107, 1591-1598

8. Shah, N. P., Tran, C., Lee, F. Y., Chen, P., Norris, D., and Sawyers, C. L. (2004) Overriding imatinib resistance with a novel ABL kinase inhibitor. Science 305, 399-401

9. O'hare, T., Shakespeare, W. C., Zhu, X., Eide, C. A., Rivera, V. M., Wang, F., Adrian, L. T., Zhou, T., Huang, W.-S., Xu, Q., Metcalf, C. A., Tyner, J. W., Loriaux, M. M., Corbin, A. S., Wardwell, S., Ning, Y., Keats, J. A., Wang, Y., Sundaramoorthi, R., Thomas, M., Zhou, D., Snodgrass, J., Commodore, L., Sawyer, T. K., Dalgarno, D. C., Deininger, M. W. N., Druker, B. J., and Clackson, T. (2009) AP24534, a pan-BCR-ABL inhibitor for chronic myeloid leukemia, potently inhibits the T315I mutant and overcomes mutation-based resistance. Cancer Cell 16, 401-412

10. Jabbour, E., Jones, D., Kantarjian, H. M., O'Brien, S., Tam, C., Koller, C., Burger, J. A., Borthakur, G., Wierda, W. G., and Cortes, J. E. (2009) Long-term outcome of patients with chronic myeloid leukemia treated with second-generation tyrosine kinase inhibitors after imatinib failure is predicted by the in vitro sensitivity of BCR-ABL kinase domain mutations. Blood 114, 2037-2043

11. Cortes, J. E., Kantarjian, H., Shah, N. P., Bixby, D., Mauro, M. J., Flinn, I., O'Hare, T., Hu, S., Narasimhan, N. I., Rivera, V. M., Clackson, T., Turner, C. D., Haluska, F. G., Druker, B. J., Deininger, M. W., and Talpaz, M. (2012) Ponatinib in refractory Philadelphia chromosome-positive leukemias. $N$ Engl J Med 367, 2075-2088

12. Apperley, J. F. (2007) Part I: mechanisms of resistance to imatinib in chronic myeloid leukaemia. Lancet Oncol 8, 1018-1029

13. Jabbour, E., Branford, S., Saglio, G., Jones, D., Cortes, J. E., and Kantarjian, H. M. (2011) Practical advice for determining the role of BCR-ABL mutations in guiding tyrosine kinase inhibitor therapy in patients with chronic myeloid leukemia. Cancer 117, 1800-1811

14. Quintas-Cardama, A., Kantarjian, H., and Cortes, J. (2009) Imatinib and beyond--exploring the full potential of targeted therapy for CML. Nat Rev Clin Oncol 6, 535-543 
15. Ernst, T., and Hochhaus, A. (2012) Chronic myeloid leukemia: clinical impact of BCR-ABL1 mutations and other lesions associated with disease progression. Semin Oncol 39, 58-66

16. Gorre, M. E., Mohammed, M., Ellwood, K., Hsu, N., Paquette, R., Rao, P. N., and Sawyers, C. L. (2001) Clinical resistance to STI-571 cancer therapy caused by BCR-ABL gene mutation or amplification. Science 293, 876-880

17. Gibbons, D. L., Pricl, S., Kantarjian, H., Cortes, J., and Quintás-Cardama, A. (2012) The rise and fall of gatekeeper mutations? The BCR-ABL1 T315I paradigm. Cancer 118 293-299

18. Nicolini, F. E., Ibrahim, A. R., Soverini, S., Martinelli, G., Muller, M. C., Hochhaus, A., Dufva, I. H., Kim, D. W., Cortes, J., Mauro, M. J., Chuah, C., Labussiere, H., Morisset, S., Roche-Lestienne, C., Lippert, E., Hayette, S., Peter, S., Zhou, W., Maguer-Satta, V., Michallet, M., Goldman, J., Apperley, J. F., Mahon, F. O., Marin, D., and Etienne, G. (2013) The BCR-ABLT315I mutation compromises survival in chronic phase chronic myelogenous leukemia patients resistant to tyrosine kinase inhibitors, in a matched pair analysis. Haematologica

19. Lovera, S., Sutto, L., Boubeva, R., Scapozza, L., Dölker, N., and Gervasio, F. L. (2012) The different flexibility of c-Src and c-Abl kinases regulates the accessibility of a druggable inactive conformation. $J$ Am Chem Soc 134 2496-2499

20. Shan, Y., Seeliger, M. A., Eastwood, M. P., Frank, F., Xu, H., Jensen, M. Ø., Dror, R. O., Kuriyan, J., and Shaw, D. E. (2009) A conserved protonation-dependent switch controls drug binding in the Abl kinase. Proc Natl Acad Sci USA 106 139-144

21. Levinson, N. M., Kuchment, O., Shen, K., Young, M. A., Koldobskiy, M., Karplus, M., Cole, P. A., and Kuriyan, J. (2006) A Src-like inactive conformation in the abl tyrosine kinase domain. PLoS Biol 4 e144

22. Aleksandrov, A., and Simonson, T. (2010) Molecular dynamics simulations show that conformational selection governs the binding preferences of imatinib for several tyrosine kinases. J Biol Chem 285 13807-13815

23. Verkhivker, G. M. (2007) In silico profiling of tyrosine kinases binding specificity and drug resistance using Monte Carlo simulations with the ensembles of protein kinase crystal structures. Biopolymers $\mathbf{8 5}$ 333-348

24. Lin, Y. L., Meng, Y., Jiang, W., and Roux, B. (2013) Explaining why Gleevec is a specific and potent inhibitor of Abl kinase. Proc Natl Acad Sci U S A 110, 1664-1669

25. Dixit, A., and Verkhivker, G. M. (2009) Hierarchical modeling of activation mechanisms in the ABL and EGFR kinase domains: thermodynamic and mechanistic catalysts of kinase activation by cancer mutations. PLoS Comput Biol 5 e 1000487

26. Dixit, A., and Verkhivker, G. M. (2011) Computational modeling of allosteric communication reveals organizing principles of mutation-induced signaling in ABL and EGFR kinases. PLoS Comput Biol 7 e1002179

27. Lee, T.-S., Ma, W., Zhang, X., Giles, F., Cortes, J., Kantarjian, H., and Albitar, M. (2008) BCR-ABL alternative splicing as a common mechanism for imatinib resistance: evidence from molecular dynamics simulations. Mol Cancer Ther 7 3834-3841

28. Lee, T.-S., Potts, S. J., and Albitar, M. (2009) Basis for resistance to imatinib in 16 BCR-ABL mutants as determined using molecular dynamics. Recent patents on anti-cancer drug discovery 4 164-173

29. Lee, T.-S., Potts, S. J., Kantarjian, H., Cortes, J., Giles, F., and Albitar, M. (2008) Molecular basis explanation for imatinib resistance of BCR-ABL due to T315I and P-loop mutations from molecular dynamics simulations. Cancer 112 1744-1753

30. Pricl, S., Fermeglia, M., Ferrone, M., and Tamborini, E. (2005) T315I-mutated Bcr-Abl in chronic myeloid leukemia and imatinib: insights from a computational study. Mol Cancer Ther 4 1167-1174

31. Zhou, T., Commodore, L., Huang, W.-S., Wang, Y., Thomas, M., Keats, J., Xu, Q., Rivera, V. M., Shakespeare, W. C., Clackson, T., Dalgarno, D. C., and Zhu, X. (2011) Structural mechanism of the 
Pan-BCR-ABL inhibitor ponatinib (AP24534): lessons for overcoming kinase inhibitor resistance. Chemical biology \&amp; drug design 77 1-11

32. Nagar, B., Bornmann, W. G., Pellicena, P., Schindler, T., Veach, D. R., Miller, W. T., Clarkson, B., and Kuriyan, J. (2002) Crystal structures of the kinase domain of c-Abl in complex with the small molecule inhibitors PD173955 and imatinib (STI-571). Cancer Res 62 4236-4243

33. Tokarski, J. S., Newitt, J. A., Chang, C. Y. J., Cheng, J. D., Wittekind, M., Kiefer, S. E., Kish, K., Lee, F. Y. F., Borzillerri, R., Lombardo, L. J., Xie, D., Zhang, Y., and Klei, H. E. (2006) The structure of Dasatinib (BMS-354825) bound to activated ABL kinase domain elucidates its inhibitory activity against imatinib-resistant ABL mutants. Cancer Res 66 5790-5797

34. Preyer, M., Vigneri, P., and Wang, J. Y. (2011) Interplay between kinase domain autophosphorylation and F-actin binding domain in regulating imatinib sensitivity and nuclear import of BCR-ABL. PLoS One 6, e17020

35. Sali, A., and Blundell, T. L. (1993) Comparative protein modelling by satisfaction of spatial restraints. Journal of Molecular Biology 234 779-815

36. Bernstein, F. C., Koetzle, T. F., Williams, G. J., Meyer, E. F., Brice, M. D., Rodgers, J. R., Kennard, O., Shimanouchi, T., and Tasumi, M. (1977) The Protein Data Bank: a computer-based archival file for macromolecular structures. Journal of Molecular Biology 112 535-542

37. Cowan-Jacob, S. W., Fendrich, G., Floersheimer, A., Furet, P., Liebetanz, J., Rummel, G., Rheinberger, P., Centeleghe, M., Fabbro, D., and Manley, P. W. (2007) Structural biology contributions to the discovery of drugs to treat chronic myelogenous leukaemia. Acta Crystallogr D Biol Crystallogr 63 8093

38. van der Spoel, D., Lindahl, E., Hess, B., Groenhof, G., Mark, A. E., and Berendsen, H. J. C. (2005) GROMACS: fast, flexible, and free. Journal of computational chemistry 26 1701-1718

39. Sorin, E. J., and Pande, V. S. (2005) Exploring the helix-coil transition via all-atom equilibrium ensemble simulations. Biophysical Journal 88 2472-2493

40. Hornak, V., Abel, R., Okur, A., Strockbine, B., Roitberg, A., and Simmerling, C. (2006) Comparison of multiple Amber force fields and development of improved protein backbone parameters. Proteins $\mathbf{6 5}$ $712-725$

41. Jorgensen, W., Chandrasekhar, J., Madura, J., Impey, R., and Klein, M. (1983) Comparison of simple potential functions for simulating liquid water. J Chem Phys $\mathbf{7 9} 926$

42. Wang, J., Wolf, R. M., Caldwell, J. W., Kollman, P. A., and Case, D. A. (2004) Development and testing of a general amber force field. Journal of computational chemistry 25 1157-1174

43. Jakalian, A., Jack, D. B., and Bayly, C. I. (2002) Fast, efficient generation of high-quality atomic charges. AM1-BCC model: II. Parameterization and validation. Journal of computational chemistry 23 1623-1641

44. Pandini, A., Fornili, A., Fraternali, F., and Kleinjung, J. (2012) Detection of allosteric signal transmission by information-theoretic analysis of protein dynamics. FASEB J 26 868-881

45. Kabsch, W., and Sander, C. (1983) Dictionary of protein secondary structure: pattern recognition of hydrogen-bonded and geometrical features. Biopolymers 22 2577-2637

46. Barrett, C. P., Hall, B. A., and Noble, M. E. M. (2004) Dynamite: a simple way to gain insight into protein motions. Acta Crystallogr D Biol Crystallogr $602280-2287$

47. Daura, X., Gademann, K., Jaun, B., Seebach, D., van Gunsteren, W. F. and Mark, A. E. (1999) Peptide folding: when simulation meets experiment. Angew. Chem. Int. Ed. 38, 236-240

48. Steinbrecher, T., Latzer, J., and Case, D. A. (2012) Revised AMBER parameters for bioorganic phosphates. J Chem Theory Comput 8, 4405-4412

49. Choi, Y., Sims, G. E., Murphy, S., Miller, J. R., and Chan, A. P. (2012) Predicting the functional effect of amino acid substitutions and indels. PLoS One 7, e46688 
50. Kumar, P., Henikoff, S., and Ng, P. C. (2009) Predicting the effects of coding non-synonymous variants on protein function using the SIFT algorithm. Nat Protoc 4, 1073-1081

51. Adzhubei, I. A., Schmidt, S., Peshkin, L., Ramensky, V. E., Gerasimova, A., Bork, P., Kondrashov, A. S., and Sunyaev, S. R. (2010) A method and server for predicting damaging missense mutations. Nat Methods 7, 248-249

52. Humphrey, W., Dalke, A., and Schulten, K. (1996) VMD -- Visual Molecular Dynamics. Journal of Molecular Graphics 14 33-38

53. Notredame, C., Higgins, D. G., and Heringa, J. (2000) T-Coffee: A novel method for fast and accurate multiple sequence alignment. Journal of Molecular Biology 302 205-217

54. Azam, M., Seeliger, M. A., Gray, N. S., Kuriyan, J., and Daley, G. Q. (2008) Activation of tyrosine kinases by mutation of the gatekeeper threonine. Nat Struct Mol Biol 15, 1109-1118

55. Griswold, I. J., MacPartlin, M., Bumm, T., Goss, V. L., O'Hare, T., Lee, K. A., Corbin, A. S., Stoffregen, E. P., Smith, C., Johnson, K., Moseson, E. M., Wood, L. J., Polakiewicz, R. D., Druker, B. J., and Deininger, M. W. (2006) Kinase domain mutants of Bcr-Abl exhibit altered transformation potency, kinase activity, and substrate utilization, irrespective of sensitivity to imatinib. Mol Cell Biol 26, 6082-6093

56. Shah, N. P. (2011) Ponatinib: targeting the T315I mutation in chronic myelogenous leukemia. Clin Adv Hematol Oncol 9 925-926

57. Manley, P. W., Cowan-Jacob, S. W., Buchdunger, E., Fabbro, D., Fendrich, G., Furet, P., Meyer, T., and Zimmermann, J. (2002) Imatinib: a selective tyrosine kinase inhibitor. Eur J Cancer 38 Suppl 5, S19-27

58. Bradeen, H. A., Eide, C. A., O'Hare, T., Johnson, K. J., Willis, S. G., Lee, F. Y., Druker, B. J., and Deininger, M. W. (2006) Comparison of imatinib mesylate, dasatinib (BMS-354825), and nilotinib (AMN107) in an N-ethyl-N-nitrosourea (ENU)-based mutagenesis screen: high efficacy of drug combinations. Blood 108, 2332-2338

59. Jabbour, E., and Kantarjian, H. (2012) Chronic myeloid leukemia: 2012 update on diagnosis, monitoring, and management. Am J Hematol 87, 1037-1045

60. Baccarani, M., Cortes, J., Pane, F., Niederwieser, D., Saglio, G., Apperley, J., Cervantes, F., Deininger, M., Gratwohl, A., Guilhot, F., Hochhaus, A., Horowitz, M., Hughes, T., Kantarjian, H., Larson, R., Radich, J., Simonsson, B., Silver, R. T., Goldman, J., Hehlmann, R., and European, L. (2009) Chronic myeloid leukemia: an update of concepts and management recommendations of European LeukemiaNet. $J$ Clin Oncol 27, 6041-6051

61. Zhou, T., Parillon, L., Li, F., Wang, Y., Keats, J., Lamore, S., Xu, Q., Shakespeare, W., Dalgarno, D., and Zhu, X. (2007) Crystal structure of the T315I mutant of AbI kinase. Chem Biol Drug Des 70, 171181

62. Quintas-Cardama, A., and Cortes, J. (2008) Therapeutic options against BCR-ABL1 T315I-positive chronic myelogenous leukemia. Clin Cancer Res 14, 4392-4399

63. Wheeler, D. L., Dunn, E. F., and Harari, P. M. (2010) Understanding resistance to EGFR inhibitorsimpact on future treatment strategies. Nat Rev Clin Oncol 7, 493-507

64. Guo, T., Agaram, N. P., Wong, G. C., Hom, G., D'Adamo, D., Maki, R. G., Schwartz, G. K., Veach, D., Clarkson, B. D., Singer, S., DeMatteo, R. P., Besmer, P., and Antonescu, C. R. (2007) Sorafenib inhibits the imatinib-resistant KITT670I gatekeeper mutation in gastrointestinal stromal tumor. Clin Cancer Res 13, 4874-4881

65. Barf, T., and Kaptein, A. (2012) Irreversible protein kinase inhibitors: balancing the benefits and risks. $J$ Med Chem 55, 6243-6262

66. Huang, W. S., Metcalf, C. A., Sundaramoorthi, R., Wang, Y., Zou, D., Thomas, R. M., Zhu, X., Cai, L., Wen, D., Liu, S., Romero, J., Qi, J., Chen, I., Banda, G., Lentini, S. P., Das, S., Xu, Q., Keats, J., Wang, F., Wardwell, S., Ning, Y., Snodgrass, J. T., Broudy, M. I., Russian, K., Zhou, T., Commodore, L., Narasimhan, N. I., Mohemmad, Q. K., Iuliucci, J., Rivera, V. M., Dalgarno, D. C., Sawyer, T. K., 
Clackson, T., and Shakespeare, W. C. (2010) Discovery of 3-[2-(imidazo[1,2-b]pyridazin-3-yl)ethynyl]4-methyl-N-\{4-[(4-methylpiperazin-1-y 1)methyl]-3-(trifluoromethyl)phenyl\} benzamide (AP24534), a potent, orally active pan-inhibitor of breakpoint cluster region-abelson (BCR-ABL) kinase including the T315I gatekeeper mutant. J Med Chem 53, 4701-4719

67. De Falco, V., Buonocore, P., Muthu, M., Torregrossa, L., Basolo, F., Billaud, M., Gozgit, J. M., Carlomagno, F., and Santoro, M. (2013) Ponatinib (AP24534) is a novel potent inhibitor of oncogenic RET mutants associated with thyroid cancer. J Clin Endocrinol Metab 98, E811-819 


\section{FIGURE LEGENDS}

Figure 1. Cartoon showing the amino acids within the BCR-ABL catalytic domain that generate hydrogen bonds with different TKIs. Structural representation of PON (brown; PDB accession number: 3OXZ) (A), IM (orange; PDB accession number: 2HYY) $(B)$ and DAS (blue; PDB accession number: 2GQG) $(C)$ fitted inside the BCR-ABL KD (top panels). In lower panels, amino acidic residues generating hydrogen bonds with each drug are highlighted with the corresponding color.

Figure 2. I360T is the only substitution that maintains BCR-ABL catalytic activity and transforming potential. A) Cell lysates from $\mathrm{Ba} / \mathrm{F} 3$ cells lentivirally transduced with HA-tagged $A B L$ constructs displaying the specified mutations were blotted using anti-HA (top panel), anti-phosphotyrosine (middle panel) or anti-Actin (bottom panel) antibodies. Wild-type (WT) and T315I constructs were used as controls. EV = empty vector. Actin levels confirmed equal protein loading in each lane. $B$ ) The same cells were also employed to perform proliferation assays for 24, 48 or 72 hours. Numbers represent average \pm standard deviations of three separate experiments performed in quadruplicates. Results are expressed as relative luminescence fold increase with cells plated at baseline arbitrarily set at $1 . C, D$ ) Identical experiments were repeated on $\mathrm{Ba} / \mathrm{F} 3$ cells transduced with FLAGtagged BCR-ABL mutants that reproduce the amino acidic substitutions generated for ABL. Anti-FLAG immunoblots confirmed protein expression of each construct. E) In vitro kinase reactions of BCR-ABL mutants were performed in the presence of increasing concentrations of the Abltide synthetic substrate. Velocity is plotted versus peptide concentrations in a Michaelis-Menten graph. KI BCR-ABL was used as negative control. Velocity values are expressed as relative luminescence fold increase, with background calculated by omitting substrate addition in the reaction. Results shown represent average \pm standard deviations of three separate experiments performed in triplicates. $F$ ) $\mathrm{K}_{\mathrm{m}}$ and $\mathrm{V}_{\max }$ values of different BCR-ABL mutants obtained from the Michaelis-Menten equation by plotting the velocity of the reactions as a function of the substrate concentration. $\mathrm{K}_{\text {cat }}$ values were generated from the equation $\mathrm{k}_{\mathrm{cat}}=\mathrm{V}_{\max } /[$ enzyme $]$.

Figure 3. I360T and I360R kinase domains display differences in correlated motions and helix- $\alpha \mathrm{C}$ dynamics compared to $\mathrm{ABL}$ wild-type. Correlation webs calculated from $50 \mathrm{~ns}$ production runs for $\mathrm{ABL}^{\mathrm{WT}}(A), \mathrm{ABL}^{\mathrm{I} 360 \mathrm{~T}}$ $(B)$ and $\mathrm{ABL}^{\mathrm{I} 360 \mathrm{R}}(C) \mathrm{KDs}$ are shown as a cartoon representation with the DFG motif indicated in red and the activation loop in blue. Residues with correlated motions are connected by a line. Only correlations greater than 0.6 were reported. The time evolution of the secondary structure elements during the molecular dynamics simulations is shown below each cartoon. The simulation time is reported on the $\mathrm{x}$-axis while the protein 
residues index is specified on the $\mathrm{y}$-axis. Residues belonging to helix- $\alpha \mathrm{C}$ are designated by a red arrow. Secondary structures were attributed with DSSP (45): $\alpha$-helixes (blue), $\beta$-sheets (red), turns (yellow), bends (green), B-bridges (black), coils (white), 3-helixes (gray).

Figure 4. Variations in hydrogen bond interactions modulate TKI efficacy on BCR-ABL ${ }^{\mathrm{I} 60 \mathrm{~T}}$. Average distances of hydrogen bond interactions between $\operatorname{PON}(A, B, C), \operatorname{IM}(D, E, F)$ or DAS $(G, H, I)$ and the indicated residues. Results were computed for BCR-ABL ${ }^{\mathrm{WT}}$ and for the T315I and I360T mutants. Top panels show time evolution of the distances between inhibitors and residues. Lines were colored according to the tint of each amino acid (blue E286, orange T315, black M318, green I360 and red D381). Bottom panels are a snapshot collected after $35 \mathrm{~ns}$ of the corresponding simulation, with inhibitor structure shown in liquorice and weak $(>3.5 \AA$, red) or strong $(<3.5 \AA$, blue) hydrogen bonds represented as dashed lines.

Figure 5. BCR-ABL ${ }^{\mathrm{I} 60 \mathrm{~T}}$ catalytic activity is suppressed by multiple TKIs. $A, B, C$ ) To calculate $\mathrm{IC}_{50}$ values for the specified TKIs, Ba/F3 cells transduced with empty vector $(\mathrm{EV})(\diamond), B C R-A B L^{W T}(\mathbf{\square}), B C R-A B L^{T 315 I}(\boldsymbol{\Delta})$ or $B C R-A B L^{I 360 T}(\bigcirc)$ were treated for 24 hours with increasing concentrations of PON, IM or DAS. Values shown are expressed as fold decrease, with untreated cells arbitrarily set at 100 (left panels). To verify BCR-ABL inhibition, cell lysates from $\mathrm{Ba} / \mathrm{F} 3$ cells expressing the indicated mutants were blotted using an antiphosphotyrosine (p-Tyr) antibody (right panels). D) Average $\mathrm{IC}_{50}$ values derived from three independent experiments performed in triplicates.

Figure 6. Ponatinib "reverts" the dynamics of the BCR-ABL ${ }^{\mathrm{T} 315 \mathrm{I}}$ catalytic domain to those of the wild-type oncoprotein. Correlation webs were calculated over $50 \mathrm{~ns}$ production runs and reported on the cartoon representation of $\mathrm{BCR}-\mathrm{ABL}^{\mathrm{WT}}(A)$ and $\mathrm{BCR}^{\mathrm{T}} \mathrm{ABL}^{\mathrm{T} 315 \mathrm{I}}(B)$, alone or complexed $(C, D)$ with PON. Only correlations greater than 0.6 were included.

Figure 7. BCR-ABL amino acidic residues E286, M318, I360 and D381 are conserved in multiple human tyrosine kinases. A) Table including the twelve known PON targets, that summarizes the position of the conserved residues in the specified protein kinases. An Alanine or a Cysteine are always found in place of BCR-ABL M318. Isoleucine is replaced by a Valine in 4 of 12 proteins. This conservative substitution does not influence the interaction with the inhibitor, since the hydrogen bond generated with PON involves the backbone oxygen of I360 and can be reproduced by the equivalent oxygen atom in the Valine backbone. B) List of 46 human tyrosine kinases displaying residues equivalent to E286, M318, I360 and D381 identified in the BCR- 
ABL KD. Isoleucine in position 360 is replaced by a Valine in 29 of 46 tyrosine kinases. The reported proteins were efficiently inhibited by PON $\left(\mathrm{IC}_{50}<20 \mathrm{nM}\right)$ in a previously published in vitro kinase assay, with the exception of the underlined kinases $\left(\mathrm{IC}_{50}>20 \mathrm{nM}\right)$. Proteins in italics have not been tested. 
Table 1. Conservative (C) and non-conservative (NC) substitutions inserted in the BCR-ABL catalytic domain

\begin{tabular}{|c|c|c|c|}
\hline AMINO ACID & ABL POSITION & (C) MUTATION & (NC) MUTATION \\
\hline Glutamic Acid (E) & 286 & Aspartic Acid (D) & Arginine (R) \\
\hline Methionine (M) & 318 & Threonine (T) & Lysine (K) \\
\hline Isoleucine (I) & 360 & Threonine (T) & Arginine (R) \\
\hline Aspartic Acid (D) & 381 & Glutamic Acid (E) & Arginine (R) \\
\hline
\end{tabular}

Letters in parenthesis refer to the corresponding single letter code for each amino acidic residue. Amino acid positions specified above are derived from the human ABL1A protein sequence (NCBI accession number NP_005148.2). However, in the manuscript we attribute these residues to the BCR-ABL sequence maintaining the ABL numbering. 
Table 2. In silico prediction of the effects of different mutations on amino acid positions 286, 318, 360 and 381

\begin{tabular}{|c|c|c|c|c|}
\hline & PROVEAN & SIFT & polyphen-2 & $\operatorname{MOE} \Delta \Delta \mathrm{G}(\mathrm{kcal} / \mathrm{mol})$ \\
\hline E286A & Deleterious & Not Tolerated & damaging & -2.49 \\
\hline E286C & Deleterious & Not Tolerated & damaging & -5.72 \\
\hline E286D & Deleterious & Not Tolerated & damaging & -1.90 \\
\hline E286F & Deleterious & Not Tolerated & damaging & -3.55 \\
\hline E286G & Deleterious & Not Tolerated & damaging & -1.39 \\
\hline E286H & Deleterious & Not Tolerated & damaging & -0.62 \\
\hline E286I & Deleterious & Not Tolerated & damaging & -3.73 \\
\hline E286K & Deleterious & Not Tolerated & damaging & 1.13 \\
\hline E286L & Deleterious & Not Tolerated & damaging & -4.52 \\
\hline E286M & Deleterious & Not Tolerated & damaging & -4.19 \\
\hline E286N & Deleterious & Not Tolerated & damaging & -2.47 \\
\hline E286P & Deleterious & Not Tolerated & damaging & -2.65 \\
\hline E286Q & Deleterious & Not Tolerated & damaging & -1.88 \\
\hline E286R & Deleterious & Not Tolerated & damaging & -3.40 \\
\hline E286S & Deleterious & Not Tolerated & damaging & -3.00 \\
\hline E286T & Deleterious & Not Tolerated & possibly damaging & -2.40 \\
\hline E286V & Deleterious & Not Tolerated & damaging & -3.93 \\
\hline E286W & Deleterious & Not Tolerated & damaging & -2.64 \\
\hline E286Y & Deleterious & Not Tolerated & damaging & -5.21 \\
\hline M318A & Deleterious & Not Tolerated & damaging & 1.26 \\
\hline M318C & Deleterious & Not Tolerated & damaging & -1.45 \\
\hline M318D & Deleterious & Not Tolerated & damaging & 4.73 \\
\hline M318E & Deleterious & Not Tolerated & damaging & 5.55 \\
\hline M318F & Deleterious & Not Tolerated & damaging & 4.01 \\
\hline M318G & Deleterious & Not Tolerated & damaging & 3.90 \\
\hline M318H & Deleterious & Not Tolerated & damaging & 9.37 \\
\hline M318I & Deleterious & Not Tolerated & damaging & -0.26 \\
\hline M318K & Deleterious & Not Tolerated & damaging & 2.79 \\
\hline M318L & Deleterious & Not Tolerated & possibly damaging & -0.01 \\
\hline M318N & Deleterious & Not Tolerated & damaging & 1.38 \\
\hline M318P & Deleterious & Not Tolerated & damaging & -0.92 \\
\hline M318Q & Deleterious & Not Tolerated & damaging & 3.55 \\
\hline M318R & Deleterious & Not Tolerated & damaging & 7.68 \\
\hline M318S & Deleterious & Not Tolerated & damaging & 2.31 \\
\hline M318T & Deleterious & Not Tolerated & damaging & 1.88 \\
\hline M318V & Deleterious & Not Tolerated & possibly damaging & -0.09 \\
\hline M318W & Deleterious & Not Tolerated & damaging & 5.32 \\
\hline M318Y & Deleterious & Not Tolerated & damaging & 2.96 \\
\hline $\mathrm{I} 360 \mathrm{~A}$ & Deleterious & Not Tolerated & damaging & 0.50 \\
\hline $\mathrm{I} 360 \mathrm{C}$ & Deleterious & Not Tolerated & damaging & -1.60 \\
\hline I360D & Deleterious & Not Tolerated & damaging & 3.05 \\
\hline $\mathrm{I} 360 \mathrm{E}$ & Deleterious & Not Tolerated & damaging & 1.85 \\
\hline
\end{tabular}




\begin{tabular}{|c|c|c|c|c|}
\hline $\mathrm{I} 360 \mathrm{~F}$ & Deleterious & Not Tolerated & damaging & 1.18 \\
\hline I360G & Deleterious & Not Tolerated & damaging & 2.71 \\
\hline $\mathrm{I} 360 \mathrm{H}$ & Deleterious & Not Tolerated & damaging & 3.30 \\
\hline $\mathrm{I} 360 \mathrm{~K}$ & Deleterious & Not Tolerated & damaging & 1.49 \\
\hline I360L & Neutral & Not Tolerated & possibly damaging & -0.03 \\
\hline $\mathrm{I} 360 \mathrm{M}$ & Deleterious & Not Tolerated & damaging & 0.38 \\
\hline $\mathrm{I} 360 \mathrm{~N}$ & Deleterious & Not Tolerated & damaging & 1.13 \\
\hline $\mathrm{I} 360 \mathrm{P}$ & Deleterious & Not Tolerated & damaging & -0.38 \\
\hline I360Q & Deleterious & Not Tolerated & damaging & 2.00 \\
\hline I360R & Deleterious & Not Tolerated & damaging & 1.83 \\
\hline $\mathrm{I} 360 \mathrm{~S}$ & Deleterious & Not Tolerated & damaging & 0.76 \\
\hline $\mathrm{I} 360 \mathrm{~T}$ & Deleterious & Not Tolerated & damaging & 1.65 \\
\hline $\mathrm{I} 360 \mathrm{~V}$ & Neutral & Tolerated & possibly damaging & -0.10 \\
\hline $\mathrm{I} 360 \mathrm{~W}$ & Deleterious & Not Tolerated & damaging & 1.51 \\
\hline $\mathrm{I} 360 \mathrm{Y}$ & Deleterious & Not Tolerated & damaging & -0.26 \\
\hline D381A & Deleterious & Not Tolerated & damaging & -2.34 \\
\hline D381C & Deleterious & Not Tolerated & damaging & -4.91 \\
\hline D381E & Deleterious & Not Tolerated & damaging & -0.36 \\
\hline $\mathrm{D} 381 \mathrm{~F}$ & Deleterious & Not Tolerated & damaging & -5.21 \\
\hline D381G & Deleterious & Not Tolerated & damaging & -1.47 \\
\hline D381H & Deleterious & Not Tolerated & damaging & -1.51 \\
\hline D381I & Deleterious & Not Tolerated & damaging & -4.73 \\
\hline D381K & Deleterious & Not Tolerated & damaging & 0.91 \\
\hline D381L & Deleterious & Not Tolerated & damaging & -4.72 \\
\hline D381M & Deleterious & Not Tolerated & damaging & -4.16 \\
\hline D381N & Deleterious & Not Tolerated & damaging & -2.58 \\
\hline D381P & Deleterious & Not Tolerated & damaging & -6.07 \\
\hline D381Q & Deleterious & Not Tolerated & damaging & -2.33 \\
\hline D381R & Deleterious & Not Tolerated & damaging & -1.69 \\
\hline D381S & Deleterious & Not Tolerated & damaging & -3.11 \\
\hline $\mathrm{D} 381 \mathrm{~T}$ & Deleterious & Not Tolerated & damaging & -2.68 \\
\hline D381V & Deleterious & Not Tolerated & damaging & -4.50 \\
\hline D381W & Deleterious & Not Tolerated & damaging & -3.26 \\
\hline D381Y & Deleterious & Not Tolerated & damaging & -4.66 \\
\hline
\end{tabular}


Table 3. Binding free energies and inhibition constants for the different TKIs bound to the simulated mutants

\begin{tabular}{lccc|ccc} 
& \multicolumn{2}{c|}{$\Delta \mathrm{G}(\mathrm{kcal} / \mathrm{mol})$} & \multicolumn{3}{c}{$\mathrm{p} K_{\mathrm{i}}$} \\
\cline { 2 - 7 } & PON & IM & DAS & PON & IM & DAS \\
\hline WT & -13.8 & -13.5 & -12.5 & 10.0 & 9.8 & 9.1 \\
T315I & -13.4 & -14.0 & -10.0 & 9.8 & 10.2 & 7.3 \\
I360T & -13.1 & -16.0 & -10.6 & 9.5 & 11.7 & 7.7
\end{tabular}

$\mathrm{p} K_{\mathrm{i}}$ are calculated as $-\log K_{\mathrm{d}}$, where $K_{\mathrm{d}}$ is the dissociation constant derived from the corresponding $\Delta \mathrm{G}$ of binding. 
A

Ponatinib
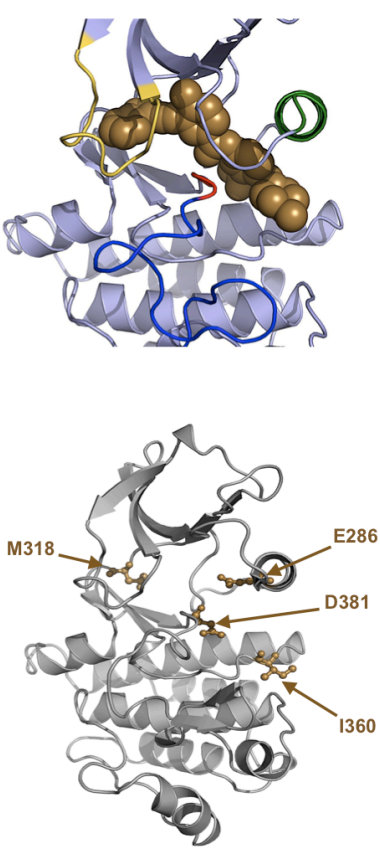

B

Imatinib
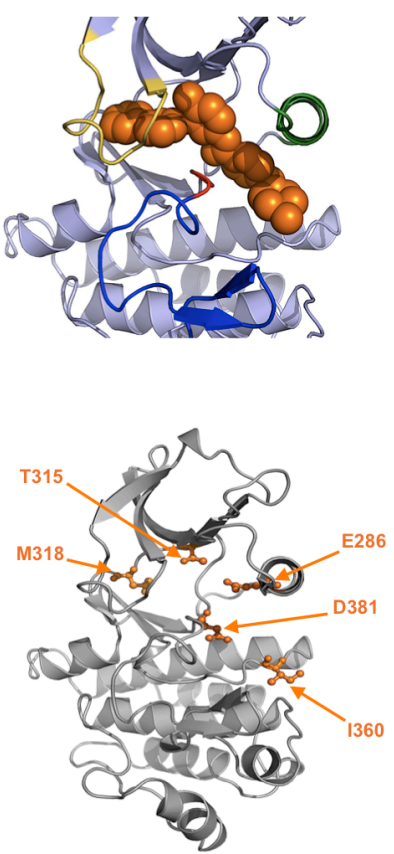

C

Dasatinib
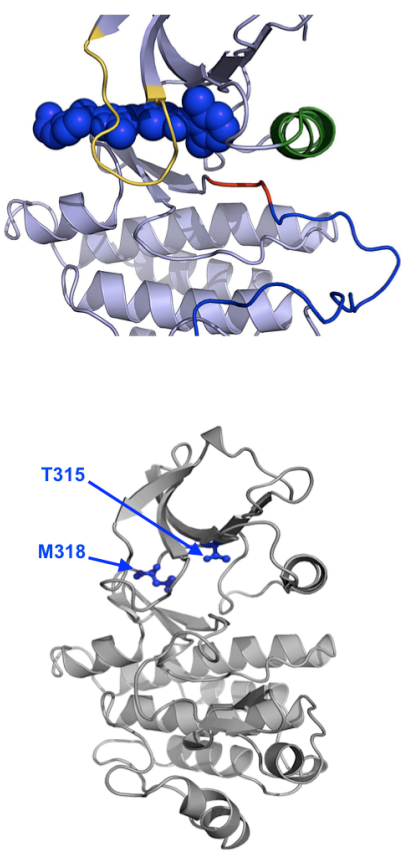

Figure 1 
A

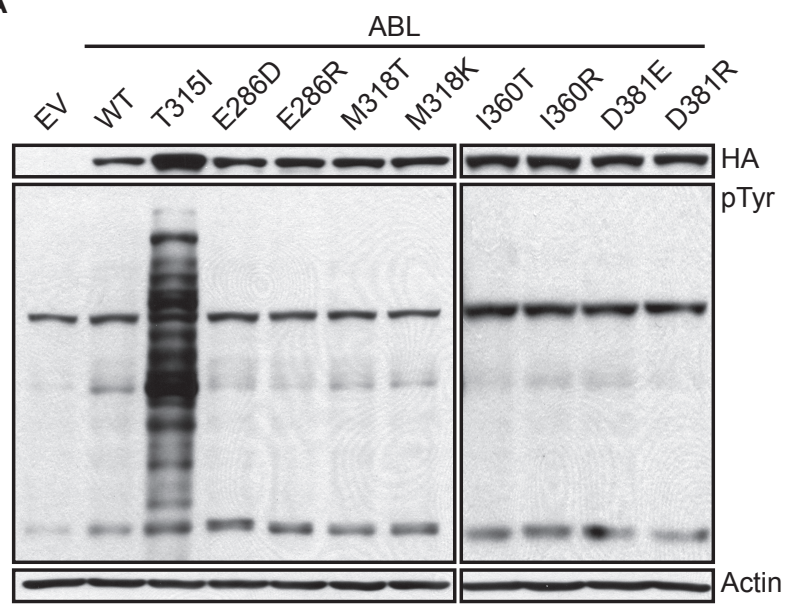

C

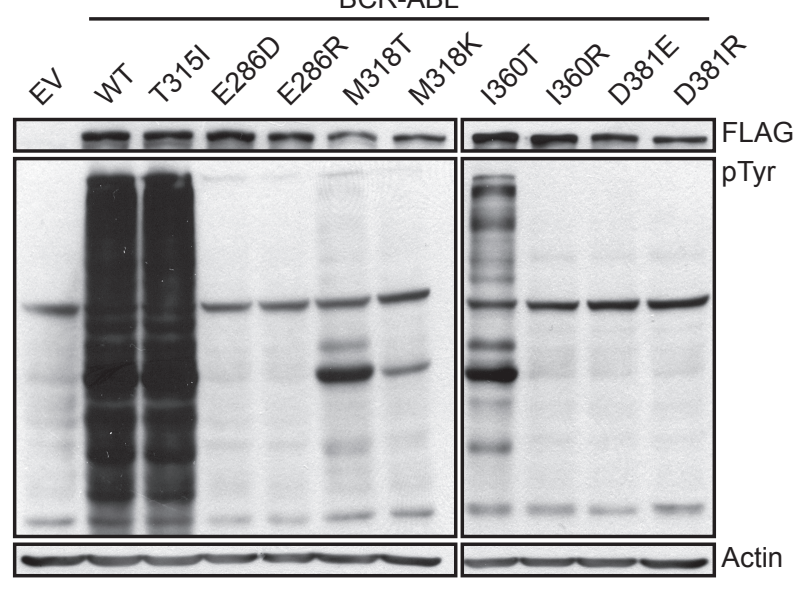

E

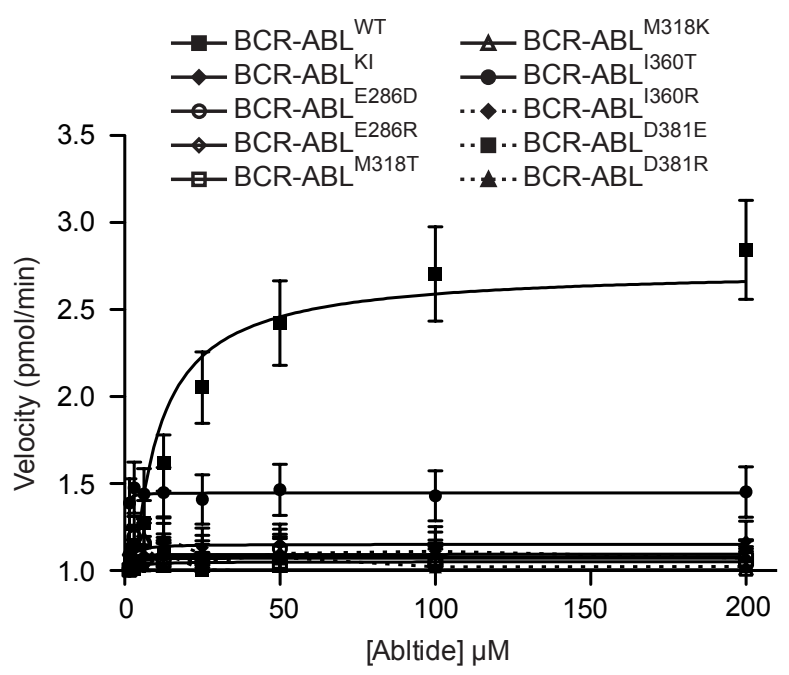

B

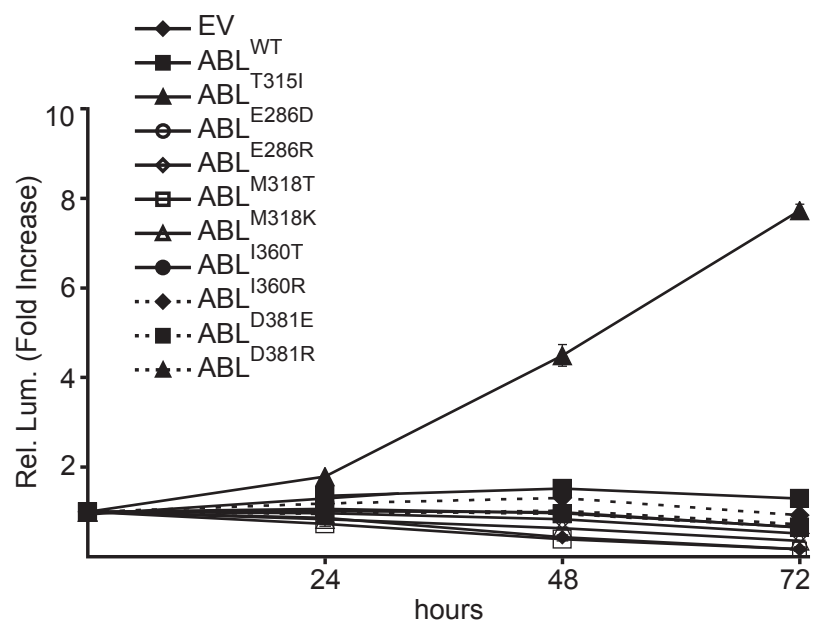

D

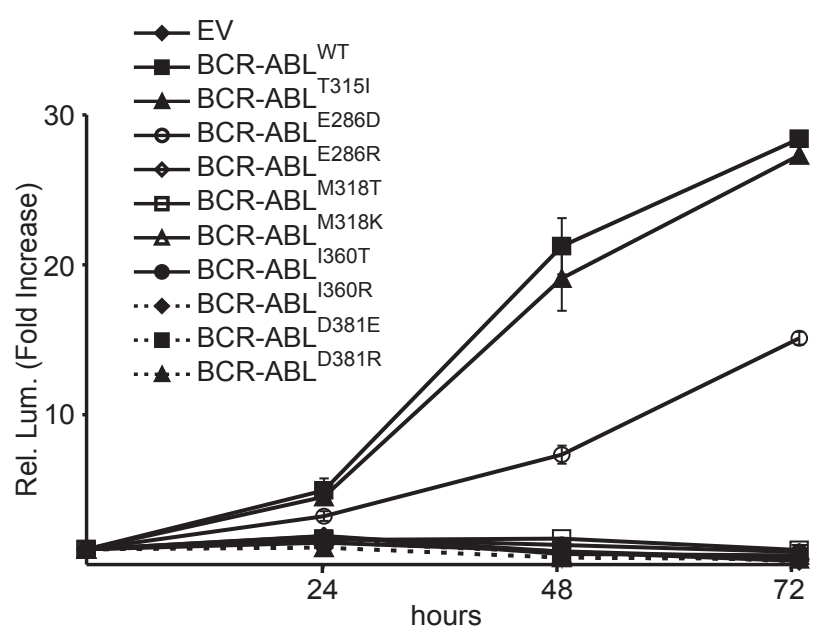

F

\begin{tabular}{lccc}
\cline { 2 - 4 } BCR-ABL $^{W T}$ & $5,650 \pm 0,959$ & $2,736 \pm 0,106$ & $0,228 \pm 0,008$ \\
BCR-ABL $^{\text {KI }}$ & $0,147 \pm 0,092$ & $1,152 \pm 0,024$ & $0,096 \pm 0,002$ \\
BCR-ABL $^{\text {E286D }}$ & $0,025 \pm 0,102$ & $1,094 \pm 0,030$ & $0,091 \pm 0,002$ \\
BCR-ABL $^{\text {E286R }}$ & 0.00 & $0,956 \pm 0,022$ & $0,079 \pm 0,002$ \\
BCR-ABL $^{\text {M318T }}$ & $0,110 \pm 0,089$ & $1,051 \pm 0,022$ & $0,087 \pm 0,002$ \\
BCR-ABL $^{\text {M318K }}$ & 0,00 & $1,079 \pm 0,028$ & $0,089 \pm 0,003$ \\
BCR-ABL $^{\text {1360T }}$ & $0,039 \pm 0,083$ & $1,448 \pm 0,030$ & $0,120 \pm 0,003$ \\
BCR-ABL $^{\text {1360R }}$ & $0,123 \pm 0,090$ & $1,080 \pm 0,022$ & $0,090 \pm 0,001$ \\
BCR-ABL $^{\text {D381E }}$ & 0,00 & $1,005 \pm 0,025$ & $0,083 \pm 0,002$ \\
BCR-ABL $^{\text {D381R }}$ & $0,041 \pm 0,095$ & $1,072 \pm 0,025$ & $0,089 \pm 0,002$
\end{tabular}


A

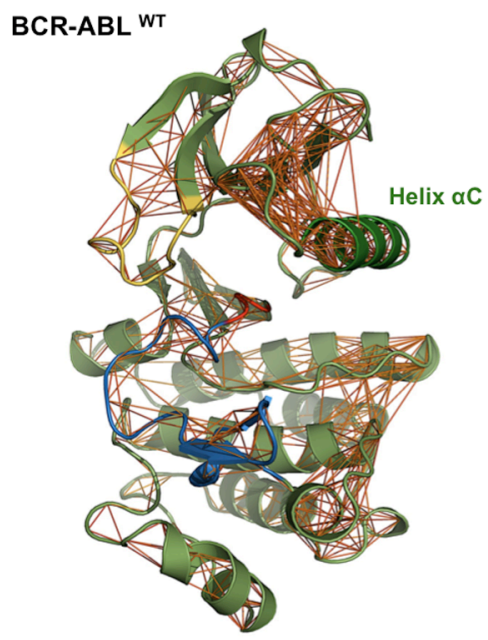

BCR-ABL WT

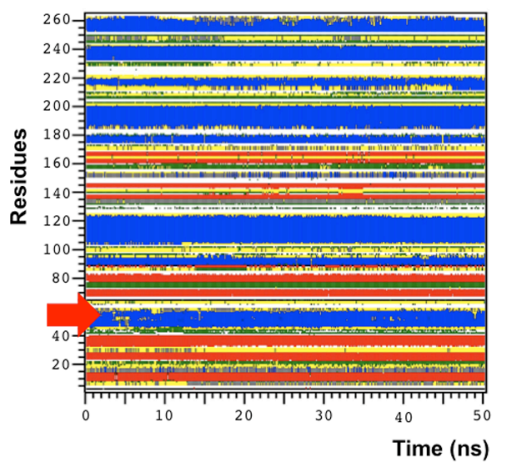

B

BCR-ABL 13600

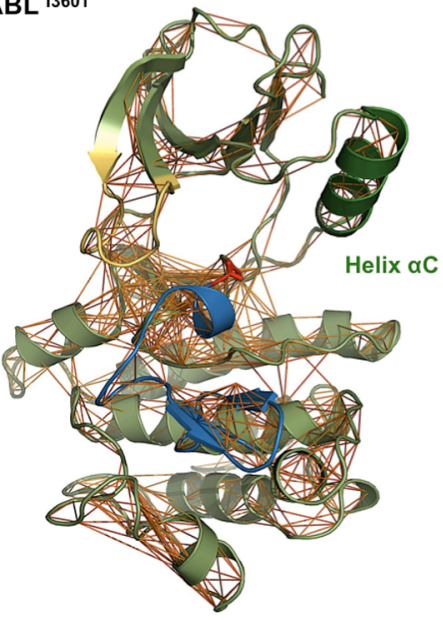

BCR-ABL 1360 T

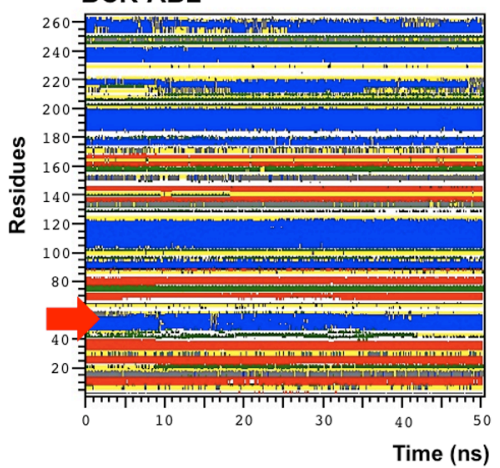

C

BCR-ABL 1360 R

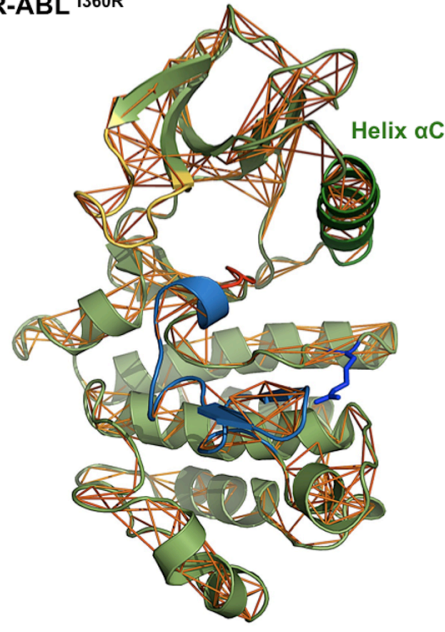

BCR-ABL 1360R

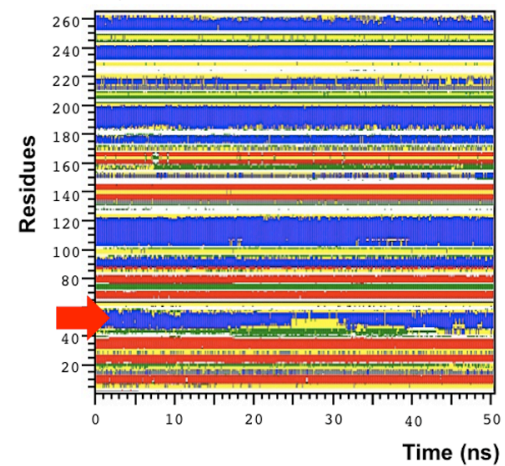

Figure 3 

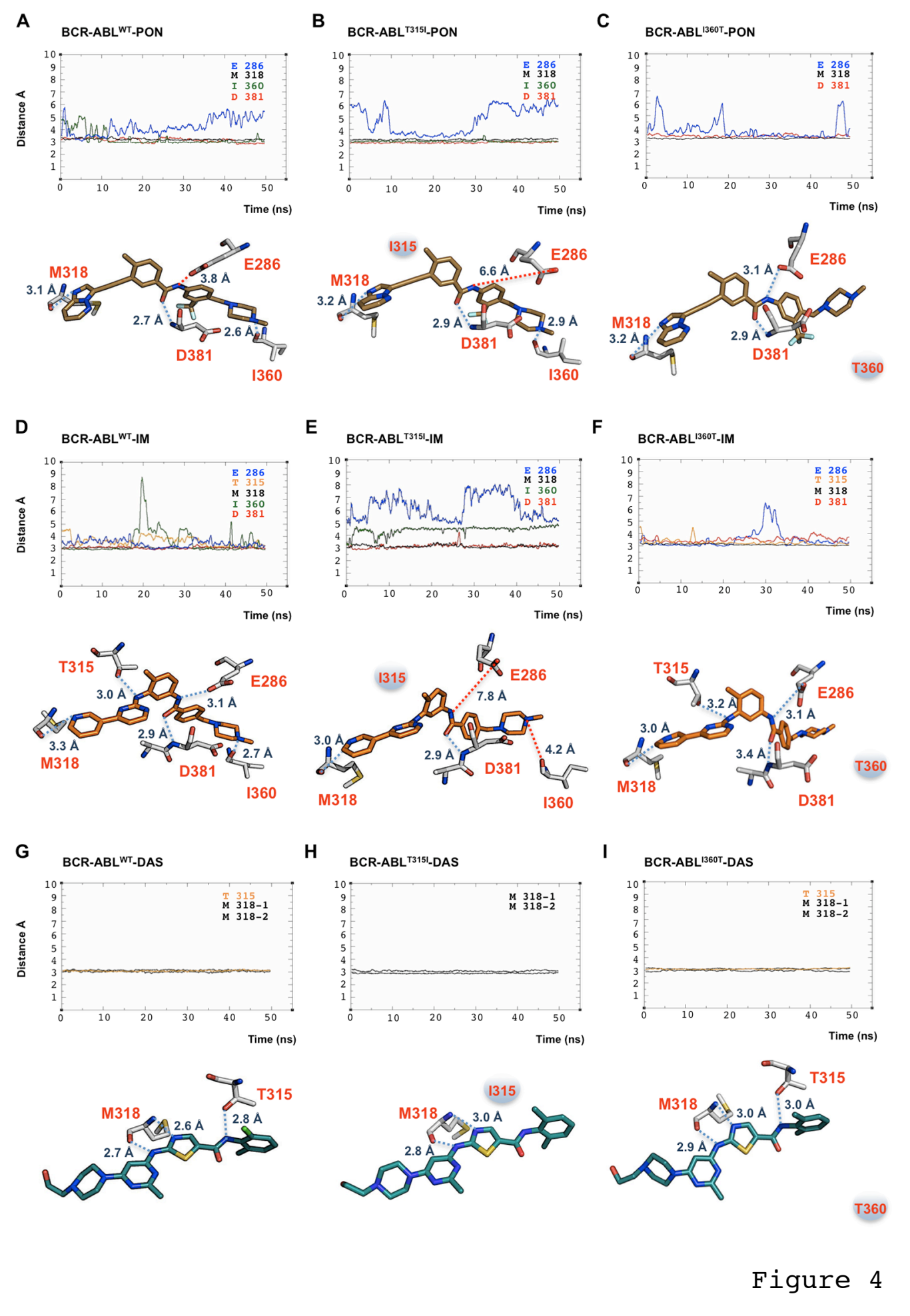
A
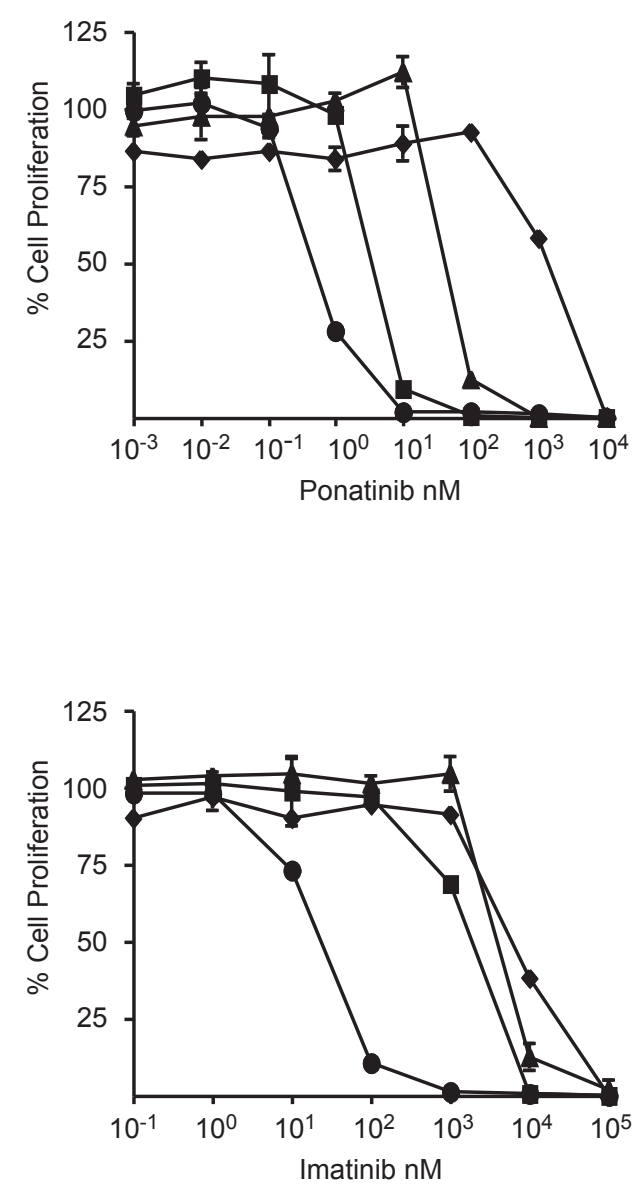

C

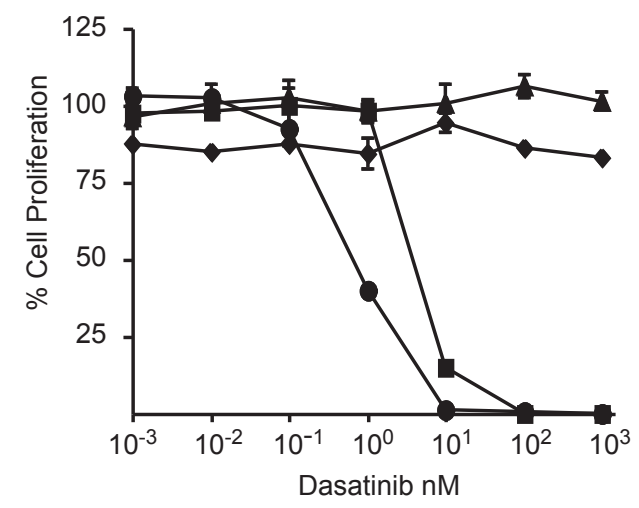

D

\begin{tabular}{lrrr} 
& \multicolumn{3}{c}{ IC50 } \\
\cline { 2 - 4 } & $\begin{array}{c}\text { Ponatinib } \\
(\text { nM })\end{array}$ & $\begin{array}{c}\text { Imatinib } \\
(n M)\end{array}$ & $\begin{array}{c}\text { Dasatinib } \\
(\text { nM })\end{array}$ \\
EV & 1131.00 & 8392.00 & $>1000.00$ \\
BCR-ABL $^{\text {WT }}$ & 3.13 & 1439.00 & 5.83 \\
BCR-ABL $^{\text {T3151 }}$ & 72.23 & 6475.00 & $>1000.00$ \\
BCR-ABL $^{\text {I360T }}$ & 0.53 & 21.15 & 0.69
\end{tabular}
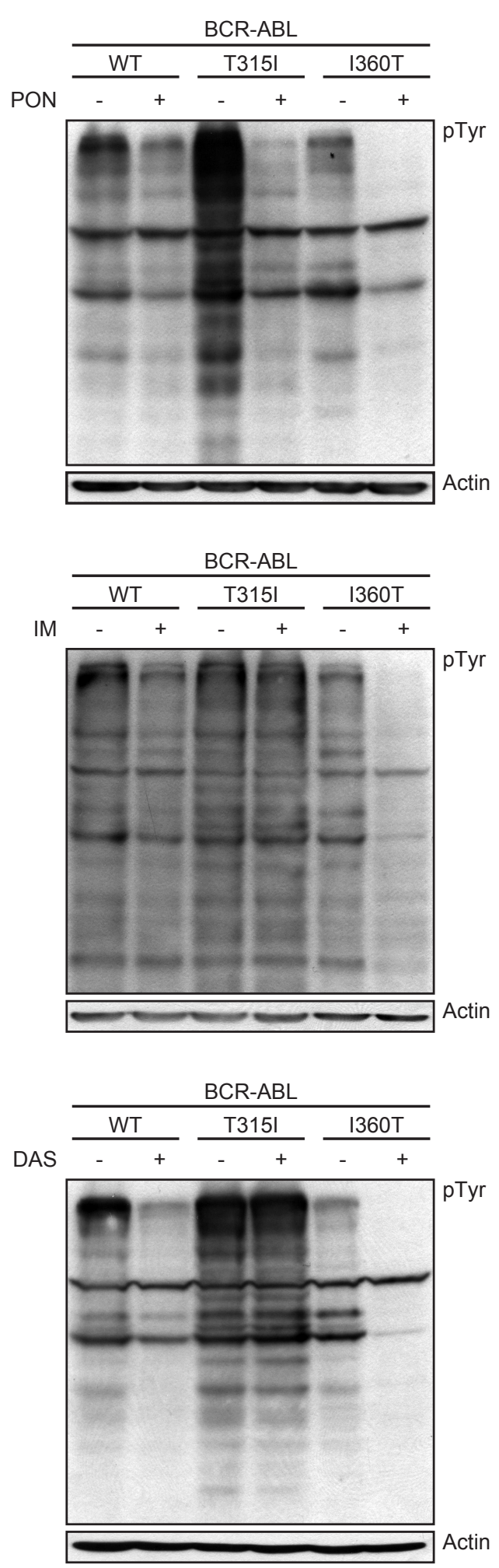

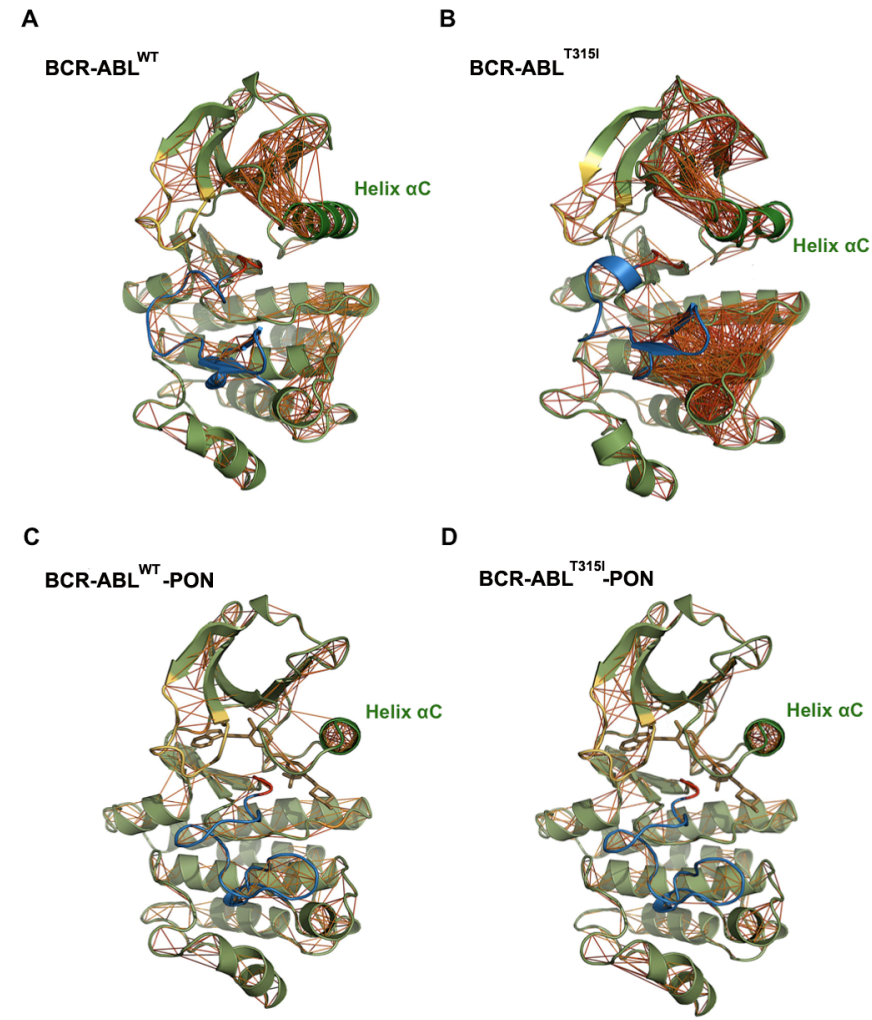

D

BCR-ABL ${ }^{\top 3151}$-PON

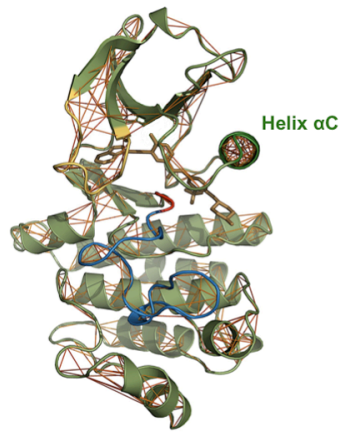

Figure 6 
A

\begin{tabular}{|l|c|c|c|c|}
\cline { 2 - 5 } \multicolumn{1}{c|}{} & E & M & I/V & D \\
\hline ABL & 286 & 318 & I 360 & 381 \\
\hline FGFR1 & 531 & A 564 & I 620 & 641 \\
FGFR2 & 534 & A 567 & I 623 & 644 \\
FGFR3 & 525 & A 558 & I 614 & 635 \\
FGFR4 & 520 & A 553 & I 609 & 630 \\
FLT3 & 661 & C 694 & V 808 & 829 \\
KIT & 640 & C 673 & I 789 & 810 \\
PDGFRa & 644 & C 677 & V 815 & 836 \\
PDGFRb & 651 & C 684 & V 823 & 844 \\
RET & 775 & A 807 & V 871 & 892 \\
VEGFR1 & 878 & C 912 & I 1019 & 1040 \\
VEGFR2 & 885 & C 919 & I 1025 & 1046 \\
VEGFR3 & 896 & C 930 & I 1034 & 1055 \\
\hline
\end{tabular}

B

\begin{tabular}{|c|c|c|c|c|}
\hline & E & $\mathbf{M}$ & $\mathrm{I} / \mathrm{V}$ & D \\
\hline ABL & 286 & 318 & 1360 & 381 \\
\hline$\underline{A L K}$ & 1167 & 1199 & | 1246 & 1270 \\
\hline ARG & 332 & 364 & 1406 & 427 \\
\hline BLK & 284 & 315 & I 357 & 378 \\
\hline$\underline{B R K}$ & 235 & 267 & 1309 & 330 \\
\hline CSK & 236 & 269 & V 311 & 332 \\
\hline$D D R 1$ & 672 & 704 & V 763 & 784 \\
\hline DDR2 & 625 & 657 & V 707 & 728 \\
\hline EGFR & 762 & 793 & V 834 & 855 \\
\hline EphA1 & 673 & 705 & V 746 & 767 \\
\hline EphA2 & 663 & 695 & V 736 & 757 \\
\hline EphA3 & 670 & 702 & V 743 & 764 \\
\hline EphA4 & 670 & 702 & V 743 & 764 \\
\hline EphA5 & 724 & 756 & V 797 & 818 \\
\hline EphA6 & 679 & 753 & V 794 & 815 \\
\hline EphA7 & 682 & 714 & V 755 & 776 \\
\hline EphA8 & 684 & 716 & V 757 & 778 \\
\hline EphB1 & 668 & 700 & V 741 & 762 \\
\hline EphB2 & 670 & 702 & V 743 & 764 \\
\hline EphB3 & 682 & 714 & V 755 & 776 \\
\hline EphB4 & 664 & 696 & V 737 & 758 \\
\hline ErbB2 & 770 & 801 & V 842 & 863 \\
\hline ErbB4 & 768 & 799 & V 840 & 861 \\
\hline FGR & 306 & 337 & I 379 & 400 \\
\hline FRK & 277 & 309 & I 351 & 372 \\
\hline FYN & 314 & 345 & I 387 & 408 \\
\hline HCK & 305 & 336 & I 378 & 399 \\
\hline$\underline{\text { IGF1R }}$ & 1050 & 1082 & V 1132 & 1153 \\
\hline$\underline{\text { INSR }}$ & 1074 & 1106 & V 1156 & 1176 \\
\hline$\underline{\text { IRR }}$ & 1030 & 1062 & V 1112 & 1133 \\
\hline$\underline{\text { ITK }}$ & 406 & 438 & I 479 & 500 \\
\hline LCK & 288 & 319 & | 361 & 382 \\
\hline LTK & 561 & 593 & 1640 & 664 \\
\hline LYN & 290 & 322 & I 364 & 385 \\
\hline$\underline{\text { MET }}$ & 1127 & 1160 & V 1201 & 1222 \\
\hline MUSK & 626 & 658 & V 722 & 743 \\
\hline$\underline{\mathrm{RON}}$ & 1131 & 1164 & V 1205 & 1226 \\
\hline$\underline{\mathrm{ROS}}$ & 1997 & 2029 & | 2076 & 2102 \\
\hline SRC & 312 & 343 & V 385 & 406 \\
\hline$\underline{\text { TEC }}$ & 413 & 445 & | 486 & 507 \\
\hline TRKA & 560 & 592 & V 647 & 668 \\
\hline TRKB & 588 & 620 & V 673 & 694 \\
\hline TRKC & 588 & 620 & V 673 & 694 \\
\hline$T X K$ & 314 & 346 & | 387 & 408 \\
\hline TYR03 & 568 & 606 & I 652 & 673 \\
\hline YES & 320 & 351 & I 393 & 414 \\
\hline Zap70 & 386 & 416 & V 458 & 479 \\
\hline
\end{tabular}

Figure 7 